\title{
Reliable and Energy-Efficient Hybrid Screen Mirroring Multicast System
}

\author{
Yunmin Go and Hwangjun Song
}

\begin{abstract}
This paper presents a reliable and energy-efficient hybrid screen mirroring multicast system for sharing high-quality real-time multimedia service with adjacent mobile devices over WiFi network. The proposed system employs overhearing-based multicast transmission scheme with Raptor codes and NACK-based retransmission to overcome well-known WiFi multicast problems such as low transmission rate and high packet loss rate. Furthermore, to save energy on mobile devices, the proposed system not only shapes the screen mirroring traffic, but also determines the target sink device and Raptor encoding parameters such as the number of source symbols, symbol size, and code rate while considering the energy consumption and processing delay of the Raptor encoding and decoding processes. The proposed system is fully implemented in Linux-based single board computers and examined in real WiFi network. Compared to existing systems, the proposed system can achieve good energy efficiency while providing a high-quality screen mirroring service.
\end{abstract}

Index Terms-Screen content, screen mirroring, WiFi, multicast, systematic raptor codes, overhearing

\section{INTRODUCTION}

C CREEN mirroring technology enables a mobile device to $\checkmark$ duplicate its screen content in real-time onto a large display device, such as monitor, TV, and projector. This technology allows the mobile user to overcome the constraints of the small display unit in a mobile device. Furthermore, screen mirroring can be applicable to various applications, such as gallery sharing, presentations, mobile streaming, and mobile gaming [1], [2], [3], [4], [5]. Because of its wide range of applications, state-of-the-art mobile devices typically offer screen mirroring functionality, and some commercial products are already available, e.g., AirPlay [6], Chromecast [7], MirrorOp [8], Splashtop [9], and Miracast [10]. In particular, Miracast, which is developed by the WiFi Alliance, aims to act like a wireless High Definition Multimedia Interface (HDMI) cable. In Miracast, the source device (i.e., the mobile device) encodes the screen content with H.264/AVC and transmits the compressed video data to the sink device (i.e., typically WiFi-enabled receiver connected to a TV or display device) using Real-Time Streaming Protocol (RTSP) and WiFi-Direct. Recently, the demand for screen content sharing among adjacent mobile devices has been increasing for conferences, lectures, etc. However, it is still challenging to provide screen mirroring for multiple adjacent devices because existing screen mirroring technologies support only one-to-one connection.

- Y. Go is with the Division of IT Convergence Engineering, Pohang University of Science and Technology, Pohang-si 790-784, Republic of Korea.E-mail: gnfservant@postech.ac.kr.

- H. Song is with the Department of Computer Science and Engineering, Pohang University of Science and Technology, Pohang-si 790-784, Republic of Korea. E-mail: hwangjun@postech.ac.kr.

Manuscript received 2 Nov. 2016; revised 16 June 2017; accepted 23 June 2017. Date of publication 0 . 0000; date of current version 0.0000.

(Corresponding author: Hwangjun Song.)

Recommended for acceptance by C.F. Chiasserini.

For information on obtaining reprints of this article, please send e-mail to: reprints@ieee.org, and reference the Digital Object Identifier below.

Digital Object Identifier no. 10.1109/TMC.2017.2721947
To handle this problem, it is necessary to enable WiFi 39 multicast for screen mirroring. Unfortunately, there are sev- 40 eral well-known problems in the WiFi multicast. One of the 41 most serious problems is unreliable packet delivery caused 42 by the absence of acknowledgment and packet retransmis- 43 sion request. Another problem is that the sender selects a 44 low transmission rate and high transmission power level 45 to deliver the data even to the farthest receiver from the 46 sender. Therefore, the existing WiFi multicast is not suitable 47 to provide a high-quality screen mirroring service, which 48 requires high video bitrate and error robustness. To solve 49 this problem and provide multicast video streaming over 50 WiFi network, some research efforts [11], [12] have been 51 devoted to overhearing and forward error correction (FEC)- 52 based multicast transmission. In this method, the sender 53 delivers the data to the target receiver using unicast trans- 54 mission while the non-target receivers overhear the unicast 55 transmission. Because the rate adaptation and MAC-layer 56 retransmission are operated by the unicast transmission 57 between the sender and the target receiver, high trans- 58 mission rate can be achieved. Moreover, FEC schemes are 59 employed to provide reliable data delivery to the non-target 60 receivers who cannot utilize the MAC-layer retransmission. 61 Recently, some fountain codes-based video streaming meth- 62 ods have been proposed to provide error-resilient multime- 63 dia services in the literature [13], [14], [15], [16], [17]. The 64 fountain codes have been successfully deployed for stream- 65 ing applications, due to their flexibility, coding efficiency, 66 and computational complexity [18].

However, it is still difficult to utilize overhearing and 68 fountain codes for screen mirroring multicast in the state- 69 of-the-art mobile devices because of their limited battery 70 capacity and computing power. It is well-known that a WiFi 71 network interface can consume approximately three times 72 the energy required to decode audio or video content [19], 73 [20]. The main reason is that the wireless network interface 74 of the mobile device maintains the active state to receive 75 continuous data during the streaming service. However, 76 
77 energy efficiency can be improved by shaping multimedia 78 traffic into periodic burst patterns [20], [21]. A number of 79 packets are collected at a time and sent together to a receiver 80 through a wireless network. In this case, the wireless net81 work interface at the receiver remains in the active state for 82 only a short period, instead of staying in the active state con83 sistently. Furthermore, when fountain codes are adopted, 84 the mobile device should perform supplementary fountain 85 encoding and decoding processes which require additional 86 energy and delay. In fact, the amounts of energy and delay 87 required for fountain encoding and decoding processes 88 change significantly according to the encoding parameters 89 of the fountain codes (e.g., code rate, symbol size, and the 90 number of source symbols) [36].

In this paper, we propose a reliable and energy-efficient hybrid screen mirroring multicast system for sharing highquality screen content among adjacent mobile devices. In the proposed system, the overhearing-based multicast scheme is employed to overcome well-known problems of the WiFi multicast. To mitigate the video quality degradation caused by packet loss, the proposed system utilizes systematic Raptor codes [22] as an FEC scheme and NACK-based retransmission scheme as an ARQ scheme for error correction. Raptor codes are a class of fountain codes [13] and a blockbased FEC scheme that provide systematic coding, flexibility, coding efficiency, and rateless codes. These characteristics are very useful for transmitting delay-sensitive data over error-prone wireless networks. The proposed system is designed to minimize energy consumption at the source device and sink devices while still providing a high-quality screen mirroring service. To achieve this goal, an energy consumption model of a WiFi network interface is derived, and then simple but effective energy consumption and delay models for Raptor encoding and decoding processes are obtained. Based on the derived models, the proposed system is designed to shape the screen mirroring traffic based on the buffer occupancy of the sink device and determine the target sink device and Raptor encoding parameters to minimize the overall energy consumption. Our main contributions are summarized below:

- Introduction of an energy consumption model of a WiFi network interface for the overhearing-based multicast scheme.

- Introduction of energy consumption and delay models for the SIMD-based Raptor encoding and decoding processes.

- Design of a target sink device and code rate determining algorithm for the overhearing-based multicast environment by taking into account the wireless network conditions and the energy consumption of WiFi network interfaces.

- Adjustment of Raptor encoding parameters such as code rate, symbol size, number of source symbols, and number of Raptor encoding blocks on the fly by considering time-varying wireless networks and energy consumption of Raptor encoding and decoding processes.

- Implementation of the entire proposed system on Linux-based single board computers and examination of the proposed system in real wireless network environments.

- Achieving spatial video quality improvement of $4.37 \mathrm{~dB}$ compared to DirCast [12] and energy savings of 39.05 percent compared to the ACK-based multi- 140 cast [44] while providing the same level of video 141 quality.

The rest of this paper is organized as follows. In Section 2, 143 we introduce related studies. Details of the proposed system 144 are presented in Section 3. Experimental results are provided 145 in Section 4, and concluding remarks are given in Section 5. 146

\section{Related Work}

So far, many research efforts have been devoted to screen 148 mirroring [2], [23], [24], [25], [26], [27]. Hsu et al. [2] com- 149 pared the performance of state-of-the-art screen mirroring 150 technologies. According to their measurements, there is no 151 single winning screen mirroring technology, and there is 152 some room for improvement through design considerations, 153 such as rate adaptation mechanisms and error resilience 154 tools. Furthermore, they implemented a rate adaptation 155 mechanism for a screen mirroring platform in [23]. Chandra 156 et al. [24] presented practical screen sharing system in 157 resource constrained environment. They developed a simple 158 mechanism to transform inter-update temporal redundancy 159 into intra-update spatial redundancy, and achieved good 160 compression rates and high screen capture rates. Zhang et al. 161 [25] conducted a measurement study on the power con- 162 sumption of Miracast. Using insights from the measurement, 163 they proposed some energy efficient mechanisms such as 164 adaptive video tail cutting, redundant codec operation 165 bypass, and least congested channel selection. In [26], Ha 166 et al. presented a frame filtering method that reduces the 167 Miracast traffic load by analyzing the dynamism of screen 168 content. Similarly, Bae et al. [27] proposed an adaptive frame 169 skipping method that analyzes the motion dynamics of 170 screen content. However, it is still challenging to provide 171 screen mirroring multicast because existing screen mirroring 172 systems are limited to unicast transmission.

To solve this problem, it is necessary to enable WiFi mul- 174 ticast for screen mirroring. To date, many research efforts 175 have focused on providing multicast media delivery system 176 over WiFi network [11], [12], [16], [17], [28], [29], [30], [31]. 177 Choi et al. [28] proposed a leader-based multicast service 178 (LBMS) to improve the reliability and efficiency of WiFi 179 multicast. Although the leader client of a multicast group 180 can send feedback frames for retransmission request and 181 rate adaptation, LBMS still cannot provide sufficient good- 182 put for high-quality video multicast. To improve the good- 183 put of a WiFi multicast, Park et al. [29] used the unicast 184 transmission to deliver the IPTV stream to a target receiver, 185 while non-target receivers overhear the unicast transmis- 186 sion. This WiFi multicast transmission method is called 187 pseudo-broadcast. Their analysis show that the pseudo- 188 broadcast achieves high transmission rate with retransmis- 189 sion and rate adaptation for only one target receiver, and it 190 cannot provide reliable packet delivery for non-target 191 receivers. In [11], Sen et al. proposed a pseudo-broadcast 192 based WiFi system for high-quality media delivery called 193 Medusa. Medusa first estimates the priority of a packet 194 according to the dependency of video decoding. Based on 195 the priority of the packet, it performs PHY rate adaptation, 196 packet order selection, and network coded retransmission. 197 Similar to Medusa, Chandra et al. [12] proposed DirCast to 198 minimize the airtime consumed by multicast traffic, DirCast 199 determines the destination client for pseudo-broadcast and 200 assigns an appropriate multicast group for a joining client. In 201 
addition, DirCast uses a proactive and adaptive FEC scheme to reduce the loss rate. Lin et al. [16] presented enhanced random early detection (ERED)-FEC mechanism at WiFi access point $(\mathrm{AP})$. With the wireless channel condition and network traffic load information, ERED-FEC calculates the FEC redundancy rate to improve the video quality without overloading the network. In [17], energy-efficient and contentaware FEC mechanism is proposed, which minimizes the overall transmission rate while satisfying the perceptual video quality requirement. There also have been research efforts to adopt the Raptor codes for WiFi multicast. Chiao et al. [30] demonstrated that the performance of systematic Raptor codes could protect a WiFi multicast streaming system inside a high-speed train. According to their analysis, the systematic Raptor code is an effective approach to recover the lost packets of a WiFi multicast. Choi et al. [31] presented a reliable video multicast scheme based on the Raptor codes and coordination between multiple APs. In the system, each AP transmits entirely or partially different Raptor encoded packets for reliable video multicast, and allocates the resource for Raptor code rate adaptation.

One of the major concerns of screen mirroring multicast technology is the amount of energy needed by the mobile device to conduct multicast transmission over the wireless network. To date, many energy-efficient wireless networking technologies [19], [32], [33], [34], [35], [36] have been proposed to improve the energy efficiency of the network interface on a mobile device with limited battery capacity. In [32], it was shown that traffic shaping using a proxy server can save more energy than adjusting the mobile device sleep time. Hoque et al. [19] presented an EStreamer to provide energy-efficient multimedia streaming service. EStreamer determines an optimal burst size and idle period length for a streaming client to allow the mobile device to reduce their energy consumption with seamless multimedia streaming. Shen et al. [33] proposed a Gaussian mixture model to reflect the video watching time of users over the Internet and developed a traffic shaping algorithm to determine the optimal buffering points during video playback based on the Gaussian mixture model. In [34], Poellabauer et al. proposed an effective approach to increase the time interval for a network interface to remain in doze mode and active mode. Go et al. [35] proposed a seamless high-quality HTTP adaptive streaming algorithm that considers wireless network conditions and the energy consumption of a mobile device with networking cost constraints over heterogeneous wireless networks. Kwon et al. [36] proposed a systematic Raptor codes-based energy-efficient multipath streaming transport protocol to support a seamless high-quality video streaming over heterogeneous wireless networks.

\section{Proposed Screen Mirroring Multicast SYSTEM}

The proposed system aims to provide a high-quality and energy-efficient screen mirroring multicast service among adjacent mobile devices over WiFi network. As mentioned earlier, systematic Raptor codes [22] and NACK-based retransmission scheme are adopted in the proposed system to recover lost packets over the error prone WiFi network. For energy saving at mobile devices, the proposed system shapes the screen mirroring traffic, and determines the target sink device and the Raptor encoding parameters based on the estimated energy consumption models of the WiFi

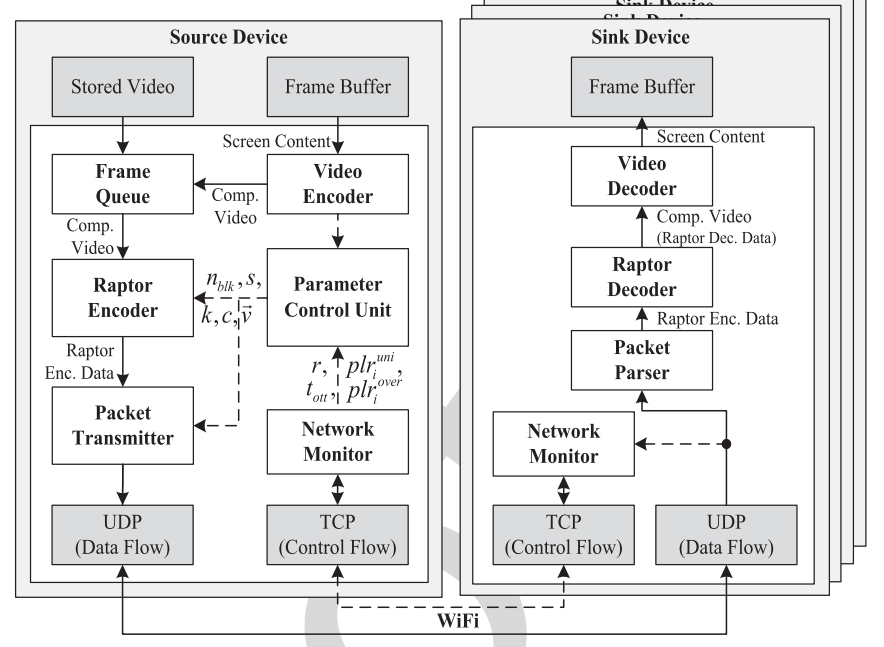

Fig. 1. Overall architecture of the proposed system.

network interface and Raptor encoding and decoding pro- 264 cesses. The overall architecture of the proposed system is 265 illustrated in Fig. 1.

As shown in Fig. 1, the proposed system is implemented 267 on both the source device and sink devices. When the mir- 268 roring content is an encoded video file stored on the source 269 device, the compressed video data are directly delivered to 270 the frame queue. When the mirroring content is the current 271 screen content on the source device, the frame buffer data of 272 the source device is moved to the video encoder for com- 273 pression, and then the compressed video data are trans- 274 ferred to the frame queue. The frame queue pushes the 275 compressed frame data to the Raptor encoder. The Raptor 276 encoder processes the frame data with the Raptor encoding 277 parameters determined by the parameter control unit, and 278 then the Raptor encoding blocks are transferred to the 279 packet transmitter. The Raptor encoding blocks are staying 280 at the packet transmitter for a specific time duration to 281 make the burst stream [20], [21]. Finally, the stream is trans- 282 mitted to multiple sink devices by the packet transmitter. 283 At this time, the proposed system employs overhearing- 284 based multicast transmission to overcome the limitations of 285 WiFi multicast. For a Raptor encoding block, the source 286 device uses a unicast transmission to a target sink device, 287 while the non-target sink devices overhear the transmitted 288 data. When the sink device receives the Raptor encoding 289 blocks, the network monitor periodically observes the WiFi 290 network conditions including transmission rate, round 291 trip time (RTT), and packet loss rate (PLR). This observed 292 information is sent to the source device using a feedback 293 message through TCP control flow, and is utilized to con- 294 sider the buffered video playback time and WiFi network 295 conditions of the sink devices. In the WiFi network, the 296 MAC-layer retransmission mechanism is supported for uni- 297 cast transmission. Owing to the lack of support, non-target 298 sink devices can only overhear the unicast transmission. 299 Thus, the PLR of overhearing is generally larger than that of 300 unicast transmission. However, it is still very difficult to 301 recover lost packets when the unexpected extreme packet 302 losses occur. In this case, the sink device requests retrans- 303 mission to the source device by transmitting NACK mes- 304 sage with information of lost packets via TCP control flow. 305

Examples of traffic shaping are presented in Fig. 2. In the 306 proposed system, a bin duration $t_{\text {bin }}$ is defined as the basic 307 
\Source data 7 Redundant data

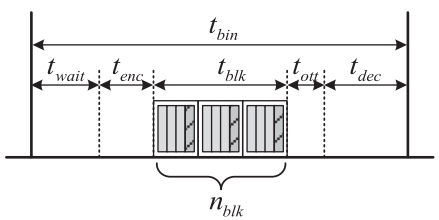

(a)

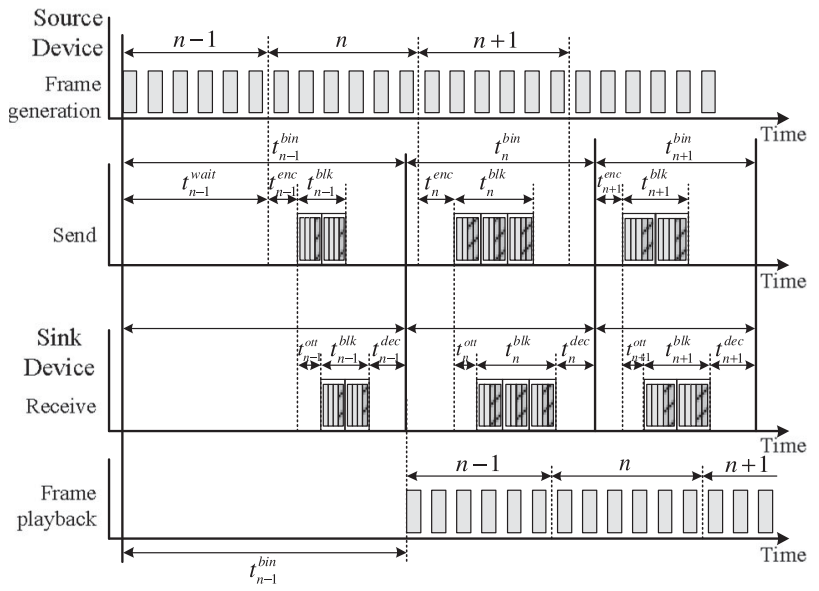

(b)

Fig. 2. Examples of traffic shaping: (a) Traffic shaping in a bin duration (b) Simple example of the traffic shaping in the source device and sink device.

interval for traffic shaping, which includes the video frame waiting time $t_{\text {wait }}$, the Raptor encoding delay $t_{\text {enc }}$, the transmission delay of Raptor encoding blocks $t_{b l k}$, the one-way trip time between the source device and sink device $t_{\text {ott }}$ (i.e., half of the maximum RTT value reported from all sink devices), and the Raptor decoding delay $t_{d e c}$. That is, $t_{b i n}$ is calculated by

$$
t_{\text {bin }}=t_{\text {wait }}+t_{\text {enc }}+t_{\text {blk }}+t_{\text {ott }}+t_{d e c} .
$$

The frame queue of the source device waits for $t_{\text {wait }}$ until a sufficient number of video frames (greater than the size of $n_{b l k}$ Raptor encoding blocks) are generated. As soon as a sufficient number of video frames arrive, they are encoded into $n_{b l k}$ Raptor encoding blocks with the same Raptor encoding parameters (symbol size $s$, the number of source symbols $k$, and code rate $c$ ), and then the Raptor encoding blocks are transmitted to the sink devices. The Raptor decoding process is triggered when the corresponding Raptor encoding block arrives at the sink device. In this paper, $c$ is calculated using $c=k / n$, where $n$ is the number of encoding symbols [36], [37]. Actually, $t_{b i n}$ is determined by the amount of pure video data transmitted during $t_{b l k}$. As $t_{b i n}$ increases, the playback delay increases while energyefficiency is improved because the WiFi network interface of the sink device can stay in the inactivate state longer, and vice versa.

\subsection{Problem Description}

In this section, we formulate the optimal problem; before presenting a detailed description, some key symbols are listed in Table 1 . Target sink device selection vector $\vec{v}$ is represented by
TABLE 1

Description of Key Symbols

\begin{tabular}{|c|c|}
\hline Symbol & Description \\
\hline$\vec{v}$ & Target sink device selection vector \\
\hline$s$ & Symbol size of Raptor encoding block \\
\hline$k$ & $\begin{array}{l}\text { Number of source symbols for Raptor } \\
\text { encoding block }\end{array}$ \\
\hline$c$ & Coder rate of Raptor encoding block \\
\hline & Number of Raptor encoding blocks \\
\hline$n_{p k t}^{s r c}$ & $\begin{array}{l}\text { Number of source data (video data) packets } \\
\text { in a Raptor encoding block }\end{array}$ \\
\hline$e_{n e t}\left(\vec{v}, n_{b l k}, s, k, c\right)$ & $\begin{array}{l}\text { Total energy consumption of the WiFi } \\
\text { network interfaces at the source and sink } \\
\text { devices during } t_{b i n}\end{array}$ \\
\hline$e_{\text {rap }}\left(\vec{v}, n_{b l k}, s, k, c\right)$ & $\begin{array}{l}\text { Total energy consumption for Raptor } \\
\text { encoding and decoding processes during } \\
t_{b i n}\end{array}$ \\
\hline$\left.n_{b l k}, s, k, c\right)$ & $\begin{array}{l}\text { Playback delay between source and sink } \\
\text { device }\end{array}$ \\
\hline$\tilde{e}_{s r c}\left(n_{b l k}, s, k\right)$ & $\begin{array}{l}\text { Energy consumption of the WiFi network } \\
\text { interface at the source device }\end{array}$ \\
\hline & $\begin{array}{l}\text { Energy consumption of the WiFi network } \\
\text { interface at the } i \text {-th sink device }\end{array}$ \\
\hline & $\begin{array}{l}\text { SIMD-based Raptor encoding energy } \\
\text { consumption for a Raptor encoding block } \\
\text { at the source device }\end{array}$ \\
\hline & $\begin{array}{l}\text { SIMD-based Raptor decoding energy con- } \\
\text { sumption for a Raptor encoding block at the } \\
i \text {-th sink device }\end{array}$ \\
\hline & Raptor encoding delay at the source device \\
\hline & $\begin{array}{l}\text { Raptor decoding delay at the } i \text {-th sink } \\
\text { device }\end{array}$ \\
\hline$E_{i}\left(v_{i}, n_{b l k}\right)$ & $\begin{array}{l}\text { Expected number of Raptor encoding } \\
\text { blocks to be decoded at the } i \text {-th sink device }\end{array}$ \\
\hline$\phi_{i}^{d e c}(s, k, c)$ & $\begin{array}{l}\text { Raptor decoding failure rate for the Raptor } \\
\text { encoding block at the } i \text {-th sink device }\end{array}$ \\
\hline$N_{\text {sink }}$ & Number of sink devices \\
\hline$S_{p k t}$ & Packet payload size \\
\hline$T_{\text {play }}^{\max }$ & $\begin{array}{l}\text { Tolerable playback delay between the } \\
\text { source and sink devices }\end{array}$ \\
\hline$\Phi_{d e c}^{\max }$ & $\begin{array}{l}\text { Tolerable maximum Raptor decoding } \\
\text { failure rate }\end{array}$ \\
\hline
\end{tabular}

$\vec{v}=\left(v_{1}, v_{2}, \ldots, v_{N_{\text {sink }}}\right)$

$v_{i}= \begin{cases}1 & \text { if the } i \text {-th sink device selected to the target sink device, } \\ 0 & \text { otherwise. }\end{cases}$

Energy consumption per packet for WiFi network inter- 343 faces and Raptor encoding and decoding processes is calcu- 344 lated by

$$
\begin{aligned}
e_{p k t} & \left(\vec{v}, n_{b l k}, s, k, c\right) \\
& =\frac{1}{n_{b l k} \cdot n_{p k t}^{s r c}} \cdot\left\{e_{n e t}\left(\vec{v}, n_{b l k}, s, k, c\right)+e_{r a p}\left(\vec{v}, n_{b l k}, s, k, c\right)\right\},
\end{aligned}
$$

where $n_{p k t}^{s r c}$ is calculated by $n_{p k t}^{s r c}=\left\lceil(s \cdot k) / S_{p k t}\right\rceil$. The playback 348 delay between source and sink device can be obtained by $\quad 349$

$$
\begin{aligned}
& t_{\text {play }}\left(\vec{v}, n_{\text {blk }}, s, k, c\right)=t_{\text {wait }}+n_{\text {blk }} \cdot t_{\text {enc }}(s, k, c) \\
& +t_{\text {blk }}+t_{\text {ott }}+\max _{1 \leq i \leq N_{\text {sink }}}\left\{E_{i}\left(v_{i}, n_{\text {blk }}\right) \cdot t_{i}^{\text {dec }}(s, k)\right\},
\end{aligned}
$$

In the proposed system, Raptor decoding is not per- 35 a formed when all original source symbols have successfully 354 arrived at the sink device. Thus, $E_{i}\left(v_{i}, n_{b l k}\right)$ is used instead 355 
of $n_{b l k}$ in Eq. (3) (Please refer to Eq. (23)). Now, we can formulate the problem to achieve our goal as follows.

Optimal Problem Formulation: Determine $\vec{v}, n_{b l k}, s, k$, and $c$ to minimize

$$
\begin{gathered}
e_{p k t}\left(\vec{v}, n_{b l k}, s, k, c\right) \\
\text { subject to } t_{\text {play }}\left(\vec{v}, n_{\text {blk }}, s, k, c\right)<T_{\text {play }}^{\max }, \\
\text { and } \max _{1 \leq i \leq N_{\text {sink }}} \phi_{i}^{\text {dec }}(s, k, c) \leq \Phi_{\text {dec }}^{\max } .
\end{gathered}
$$

Eq. (4) implies that the video frame of the source device should arrive within $T_{\text {play }}^{\max }$ in order to avoid buffer underflows at the sink devices, and Eq. (5) indicates that the source device generates sufficient number of redundant packets to support reliable screen mirroring multicast service for all sink devices (Please refer to Eq. (27)).

In fact, the control parameters of the optimal problem formulation (i.e., $\vec{v}, n_{b l k}, s, k$, and $c$ ) are tightly coupled with each other. For example, $\vec{v}$ and $c$ are strongly related to the amount of packets to be transmitted to the sink devices for the successful Raptor decoding. Furthermore, $\vec{v}$ and $c$ depend on both $s$ and $k$ because they are tightly coupled with the robustness of the Raptor encoding block. Hence, it is very difficult to obtain an optimal solution in real-time. In the proposed system, to obtain a feasible solution with a low computational complexity for real-time processing, the above problem is divided into two sub-problems: the target sink device and code rate determining problem, and the Raptor encoding parameter selection problem. The first sub-problem determines $\vec{v}$ and $c$ simultaneously because the channel states of overhearing sink devices strongly depend on which sink device is selected as a target sink device, and the code rate should be adjusted according to the corresponding channel states. Then, the second sub-problem selects Raptor encoding parameters $\left(n_{b l k}, s\right.$, and $k$ ) in a bin duration with the predetermined $\vec{v}$ and $c$. The feasible solution is obtained by performing the two algorithms iteratively.

We first describe the target sink device and code rate determining problem. The proposed system determines the target sink device and code rate to minimize the number of redundant packets for Raptor encoding blocks and the number of retransmitted packets for unexpected packet losses. In fact, the target sink device selection vector $\vec{v}$ and code rate $c$ affect the energy consumption of mobile devices because $\vec{v}$ and $c$ are strongly related to the number of redundant packets and retransmitted packets. The number of redundant packets and retransmitted packets depend on $\vec{v}$ since the number of received packets at each sink device depends on the selected target sink device. Moreover, when $c$ decreases (i.e., the number of redundant packets increases), the number of sink devices which can recover lost packets without retransmission gradually increase, but unnecessary redundant packets can only be received at some sink devices. On the other hand, when $c$ increases (i.e., the number of redundant packets decreases), the amount of retransmitted packets may increase because the number of redundant packets required for successful Raptor decoding may not be sufficient. To achieve our goal, we first define the number of unnecessary redundant packets $n_{p k t}^{u n r e}(\vec{v}, c)$ as follows.

$$
n_{p k t}^{\text {unre }}(\vec{v}, c)=\sum_{i=1}^{N_{\text {sin } k}} \max \left\{n_{i}^{\text {recv }}\left(v_{i}, c\right)-n_{p k t}^{\min }, 0\right\},
$$

$$
n_{i}^{\text {recv }}\left(v_{i}, c\right)=n_{p k t}^{b l k} \cdot\left[1-\left\{v_{i} \cdot p l r_{i}^{u n i}+\left(1-v_{i}\right) \cdot p l r_{i}^{\text {over }}\right\}\right],
$$

$$
n_{p k t}^{\min }=\left\lceil\frac{s \cdot k \cdot(1+\delta(k))}{S_{p k t}}\right\rceil,
$$

$$
n_{p k t}^{b l k}=\left\lceil\frac{s \cdot k}{c \cdot S_{p k t}}\right\rceil
$$

where $n_{i}^{\text {recv }}\left(v_{i}, c\right)$ is the number of successfully received 425 packets at the $i$ th sink device, $n_{p k t}^{\min }$ is the minimum number 426 of packets required for successful Raptor decoding, $\delta(k)$ is 427 the minimum symbol overhead, $n_{p k t}^{b l k}$ is the number of pack- 428 ets in a Raptor encoding block, and $p l r_{i}^{u n i}$ and $p l r_{i}^{\text {over }}$ are the 429 PLR of unicast transmission and the PLR of overhearing at 430 the $i$ th sink device, respectively. The number of retransmit- 431 ted packets $n_{p k t}^{r e t r}(\vec{v}, c)$ is calculated by

$$
\begin{gathered}
n_{p k t}^{\text {retr }}(\vec{v}, c)=\sum_{i=1}^{N_{\text {sink }}} n_{i}^{\text {lost }}\left(v_{i}, c\right), \\
n_{i}^{\text {lost }}\left(v_{i}, c\right)=\left\{\begin{array}{cl}
n_{i}^{\text {recv }}\left(v_{i}, c\right) & \text { if } n_{i}^{\text {recv }}\left(v_{i}, c\right)<n_{p k t}^{\min }, \\
0 & \text { otherwise, }
\end{array}\right.
\end{gathered}
$$
435

where $n_{i}^{\text {lost }}\left(v_{i}, c\right)$ is the number of lost packets at the $i$ th sink 438 device. Now, we can formulate the target sink device and 439 code rate determining problem to determine $\vec{v}$ and $c$ as 440 follows.

Sub-Problem Formulation 1. Target Sink Device and Code 442 Rate Determining Problem: Determine $\vec{v}$ and $c$ to minimize 443 the cost function $n_{p k t}^{c o r}(\vec{v}, c)$

$$
\begin{gathered}
n_{p k t}^{\text {unre }}(\vec{v}, c)+n_{p k t}^{r e t r}(\vec{v}, c) \\
\text { subject to } 0<c \leq 1, \\
\text { and } \max _{1 \leq i \leq N_{\text {sink }}} \phi_{i}^{\text {dec }}(s, k, c) \leq \Phi_{d e c}^{\max } .
\end{gathered}
$$

Finally, the above optimal problem formulation can be 451 simplified as follows with the determined $\vec{v}$ and $c$ from the 452 first sub-problem.

Sub-Problem Formulation 2. Encoding Parameters Selection 454 Problem: Determine $n_{b l k}, s$, and $k$ to minimize the cost func- 455 tion $e_{p k t}^{s e l}\left(n_{b l k}, s, k\right)$ with the given $\vec{v}$ and $c$

\section{6}

$$
\begin{aligned}
& \frac{1}{n_{b l k} \cdot n_{p k t}^{\text {src }}} \cdot\left\{e_{\text {net }}\left(n_{\text {blk }}, s, k\right)+e_{\text {rap }}\left(n_{\text {blk }}, s, k\right)\right\} \\
& \text { subject to } t_{\text {play }}\left(\vec{v}, n_{\text {blk }}, s, k, c\right)<T_{\text {play }}^{\max } .
\end{aligned}
$$

Actually, when the target sink device changes, fairness 458 problem in the performance at sink devices may occur. In 461 general, non-target sink devices require more redundant 462 packets and retransmission packets than the target sink 463 device since they only overhear the unicast transmission. 464 In addition, the possibility of performing Raptor decoding 465 at non-target sink devices is greater than that of target sink 466 device. Therefore, non-target sink devices may consume 467 more energy than the target sink device. However, it is very 468 difficult to solve the fairness problem in the performance at 469 sink devices because their battery status or power supply 470 status can be different each other. Although the fairness 471 problem in the performance of sink devices is very impor- 472 tant, we will handle this problem in the future work because 473 it is beyond the scope of this paper. 


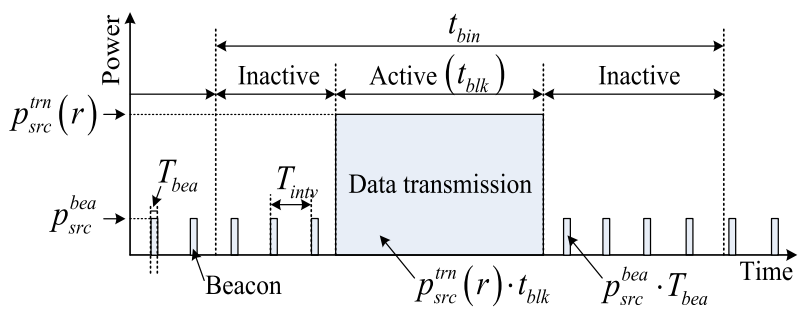

(a)

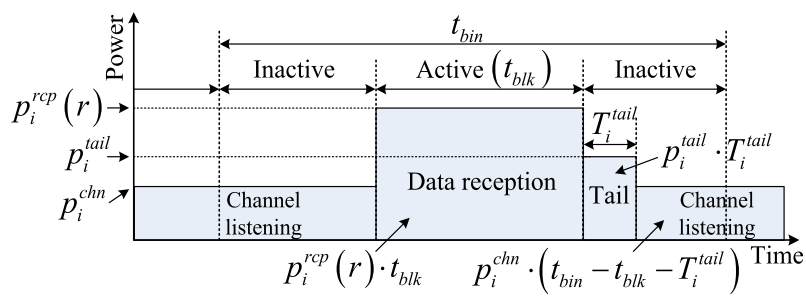

(b)

Fig. 3. Energy consumption patterns of WiFi network interfaces: (a) Source device and (b) sink device.

To solve the above problem with low computational complexity for real-time processing, we utilize the modelbased estimation method.

\subsection{Energy Consumption Model and Delay Model}

In this section, we propose an energy consumption model of the WiFi network interface and energy consumption models and delay models for Raptor encoding and decoding processes.

\subsubsection{Energy Consumption Model for WiFi Network Interface}

The general energy consumption patterns of the WiFi network interfaces at the source device and sink devices are presented in Fig. 3. Because the source device behaves like an AP, it periodically broadcasts beacon messages to the sink devices during the inactive state. The sink devices constantly listen to the wireless channel to overhear unicast transmission. When there is data to be received from the source device, the target sink device requests the data from the source device. Now, the source device transmits data using unicast transmission to the target device, and the non-target devices immediately overhear these data. After completing the data transmission, the source device and target sink device enter into the inactive state if there is no additional data for tail time [38]. However, non-target devices immediately go into the inactive state as soon as the data transmission is finished. Since only the active state (data transmission and reception) and inactive state (beacon transmission, tail, and channel listening) are considered in this paper, the energy consumption of the WiFi network interfaces during $t_{b i n}$ is the sum of the shaded areas in Fig. 3. For $n_{b l k}$ blocks encoded with the given $s, k$, and $c$, the energy consumption of the WiFi network interface at the source device and the energy consumption of the WiFi network interface at the $i$ th sink device can be modeled as follows.

$$
\begin{gathered}
\tilde{e}_{s r c}\left(n_{b l k}, s, k\right)=p_{s r c}^{t r n}(r) \cdot t_{b l k}+p_{s r c}^{\text {bea }} \cdot\left(t_{b i n}-t_{b l k}\right) \cdot\left(T_{\text {bea }} / T_{i n t v}\right), \\
\tilde{e}_{i}^{\text {sink }}\left(n_{b l k}, s, k\right)=p_{i}^{r c p}(r) \cdot t_{b l k}+p_{i}^{\text {tail }} \cdot T_{i}^{\text {tail }}+p_{i}^{c h n} \cdot\left(t_{b i n}-t_{b l k}-T_{i}^{\text {tail }}\right),
\end{gathered}
$$

where $r$ is the data transmission rate, $p_{s r c}^{t r n}(r)$ is the data trans- 514 mission power of the source device, $t_{b l k}$ is the transmission 515 delay of Raptor encoding blocks (i.e., $\left.\left(n_{b l k} \cdot s \cdot k\right) /(c \cdot r)\right), p_{s r c}^{b e a} 516$ is the beacon transmission power, $T_{\text {bea }}$ is the beacon trans- 517 mission duration, $T_{\text {intv }}$ is the interval of the beacon transmis- 518 sion, $p_{i}^{r c p}(r)$ is the data reception power of the $i$ th sink device, 519 $p_{i}^{\text {tail }}$ is the tail power of the $i$ th sink device, $p_{i}^{c h n}$ is the channel 520 listening power of the $i$ th sink device, and $T_{i}^{\text {tail }}$ is the tail time 521 of the $i$ th sink device. $p_{s r c}^{t r n}(r)$ and $p_{i}^{r c p}(r)$ are calculated using 522 a simple linear model [38] as follows.

$$
\begin{gathered}
p_{s r c}^{t r n}(r)=\beta_{s r c} \cdot r+\gamma_{s r c}, \\
p_{i}^{r c p}(r)=\beta_{i}^{s i n k} \cdot r+\gamma_{i}^{\text {sink }},
\end{gathered}
$$

where $\beta_{\text {src }}$ and $\gamma_{\text {src }}$ are the power model parameters of the 529 source device, and $\beta_{i}^{\text {sink }}$ and $\gamma_{i}^{\text {sink }}$ are the power model param- 530 eters of the $i$ th sink device $\left(\beta_{s r c}, \gamma_{s r c}, \beta_{i}^{s i n k}, \gamma_{i}^{\text {sink }}>0\right)$. In gen- 531 eral, the WiFi network interface stays in the tail state with 532 steady power consumption for a period after the data transfer 533 is completed in order to reduce signaling overhead and 534 latency [38]. The tail time is set by the device manufacturer. 535

Finally, the total energy consumption of WiFi network 536 interfaces during $t_{b i n}$ at all devices can be calculated using 537 $\tilde{e}_{s r c}\left(n_{b l k}, s, k\right)$ and $\tilde{e}_{i}^{s i n k}\left(n_{b l k}, s, k\right)$ as follows.

$$
\tilde{e}_{n e t}\left(n_{b l k}, s, k\right)=\tilde{e}_{s r c}\left(n_{b l k}, s, k\right)+\sum_{i=1}^{N_{s i n k}} \tilde{e}_{i}^{s i n k}\left(n_{b l k}, s, k\right) .
$$

\subsubsection{Energy Consumption and Delay Model for Raptor 542 Encoding/Decoding Processes \\ 543}

For Raptor encoding and decoding processes, the XOR 544 operation is the most dominant process [36], [37]. Thus, the 545 energy consumption for Raptor encoding and decoding 546 processes can be predicted based on the amount of XORed 547 bytes, which is calculated by multiplying the symbol size by 548 the number of symbol-level XOR operations. In the pro- 549 posed system, we implemented Raptor codes using a single 550 instruction multiple data (SIMD) technology [39] to improve 551 the performance of the Raptor encoding and decoding pro- 552 cesses. The SIMD is a well-known parallel processing tech- 553 nology that enables the parallel processing of multiple data 554 with a single instruction, e.g., matrix summation and multi- 555 plication. Since the performance of the Raptor codes in the 556 proposed system is affected by the SIMD-based implemen- 557 tation, we derive the SIMD-based energy consumption and 558 delay models. The SIMD-based Raptor encoding energy 559 consumption for a Raptor encoding block $\tilde{e}_{e n c}(s, k, c)$ and 560 Raptor decoding energy consumption for a Raptor encoding 561 block at the $i$ th sink device $\tilde{e}_{i}^{\operatorname{dec}}(s, k)$ can be represented by 562

$$
\begin{gathered}
\tilde{e}_{e n c}(s, k, c)=\rho_{\text {enc }}(s) \cdot\left\{s \cdot n_{\text {xor }}^{e n c}(k, c)\right\}^{\mu_{e n c}(s)}, \\
\tilde{e}_{i}^{\operatorname{dec}}(s, k)=\rho_{i}^{\operatorname{dec}}(s) \cdot\left\{s \cdot n_{\text {xor }}^{\operatorname{dec}}(k)\right\}^{\mu_{i}^{d e c}(s)},
\end{gathered}
$$

where $n_{x o r}^{e n c}(k, c)$ is the total number of symbol-level XOR 568 operations required for the Raptor encoding, $n_{x o r}^{\text {dec }}(k)$ is the 569 total number of symbol-level XOR operations required for 570 the Raptor decoding, $\rho_{\text {enc }}(s)$ and $\mu_{\text {enc }}(s)$ are the energy coeffi- 571 cients for the Raptor encoding at the source device, and 572 $\rho_{i}^{\operatorname{dec}}(s)$ and $\mu_{i}^{\operatorname{dec}}(s)$ are the energy coefficients for the Raptor 573 
decoding at the $i$ th sink device. The energy coefficients for the Raptor encoding and decoding processes are related to the computing power of the mobile device. These coefficients can be obtained by using the curve fitting method from the measured energy consumption of the Raptor encoding and decoding processes. In this paper, we adopt the LevenbergMarquardt algorithm [46], which is a well-known curve fitting method for non-linear functions. Consequently, for $n_{b l k}$ Raptor encoding blocks, the total energy consumption of Raptor encoding and decoding processes $\tilde{e}_{\text {rap }}\left(n_{b l k}, s, k\right)$ can be calculated using $\tilde{e}_{e n c}(s, k, c)$ and $\tilde{e}_{i}^{d e c}(s, k)$ as follows.

$$
\tilde{e}_{r a p}\left(n_{b l k}, s, k\right)=n_{b l k} \cdot \tilde{e}_{e n c}(s, k, c)+\sum_{i=1}^{N_{\text {sink }}} E_{i}\left(v_{i}, n_{b l k}\right) \cdot \tilde{e}_{i}^{d e c}(s, k),
$$

$$
E_{i}\left(v_{i}, n_{b l k}\right)=\left\lceil n_{b l k} \cdot\left\{v_{i} \cdot p l r_{i}^{u n i}+\left(1-v_{i}\right) \cdot p l r_{i}^{\text {over }}\right\}\right\rceil
$$

Similarly, the SIMD-based Raptor encoding delay $\tilde{t}_{\text {enc }}(s, k, c)$ and Raptor decoding delay $\tilde{t}_{i}^{d e c}(s, k)$ can be estimated based on the amount of XORed bytes, that is,

$$
\begin{gathered}
\tilde{t}_{\text {enc }}(s, k, c)=\sigma_{\text {enc }}(s) \cdot s \cdot n_{\text {xor }}^{e n c}(k, c), \\
\tilde{t}_{i}^{\operatorname{dec}}(s, k)=\sigma_{i}^{\operatorname{dec}}(s) \cdot s \cdot n_{\text {xor }}^{\text {dec }}(k),
\end{gathered}
$$

where $\sigma_{\text {enc }}(s)$ denotes the delay coefficients for Raptor encoding, and $\sigma_{i}^{\text {dec }}(s)$ denotes the delay coefficients for Raptor decoding at the $i$ th sink device. The delay coefficients for the Raptor encoding and decoding processes are obtained by using the least square solution [47]. In the proposed system, all coefficients of energy models and delay models are measured and embedded at each source and sink device before connection is set up. Coefficients of sink device, such as $\rho_{i}^{d e c}(s), \mu_{i}^{d e c}(s)$, and $\sigma_{i}^{d e c}(s)$, are provided to the source device during the connection setup process.

In fact, the energy consumption of the proposed system and the coefficients of the energy models are strongly related to the hardware specification of the mobile device. Hence, it is very difficult to find the typical coefficients of the energy models. But several device manufacturers provide the power profile to estimate the device energy consumption [49]. If the power profile is offered by device manufacturer, then we can approximately calculate the coefficients of the energy models.

\subsection{Parameter Determining Algorithm}

In this section, we present the parameter determining algorithm to obtain a feasible solution. First, the target sink device and code rate determining algorithm is studied. Then, the Raptor encoding parameter selection algorithm is described in detail.

\subsubsection{Target Sink Device and Code Rate Determining Algorithm}

We provide the determining algorithm for $\vec{v}$ and $c$. In the target sink device and code rate determining problem, when the target sink device is fixed, the solution candidates of $c$ can be obtained by calculating the code rates which can achieve a successful Raptor decoding at a certain sink device. Thus, the optimal solution of $\vec{v}$ and $c$ that minimizes the given cost function $n_{p k t}^{c o r}(\vec{v}, c)$ can be easily obtained by conducting a full search among all possible candidates of $c 632$ for all sink devices. Details of the target sink device and 633 code rate determining algorithm are presented below.

Step 0) Empty the candidate parameter set $P_{c n d}^{t c}$.

Step 1) Set the candidate target sink device selection vector 636 $\vec{v}_{\text {cnd }}$ by selecting the $j$ th sink device as a target sink 637 device $\left(1 \leq j, m \leq N_{\text {sink }}\right)$.

$$
\vec{v}_{c n d}=\left(v_{1}^{c n d}, v_{2}^{c n d}, \ldots, v_{N_{s i n k}}^{\text {cnd }}\right), v_{m}^{\text {cnd }}= \begin{cases}1 & \text { if } j=m \\ 0 & \text { otherwise }\end{cases}
$$

Step 2) Calculate code rate candidate $c_{\text {cnd }}$ for the $m$ th sink 642 device $\left(1 \leq m \leq N_{\text {sink }}\right)$.

$$
\begin{aligned}
c_{c n d} & =\frac{k}{k_{p k t} \cdot n_{m}^{\text {trs }}}, k_{p k t}=\left\lfloor\frac{S_{p k t}}{s}\right\rfloor, \\
n_{m}^{\text {trs }} & =\left\lceil\frac{n_{p k t}^{\min }}{1-\left\{v_{m}^{\text {cnd }} \cdot p l r_{m}^{\text {uni }}+\left(1-v_{m}^{\text {cnd }}\right) \cdot p l r_{m}^{\text {over }}\right\}}\right\rceil,
\end{aligned}
$$

where $k_{p k t}$ is the number of symbols in a packet, and 646 $n_{m}^{\text {trs }}$ is the minimum number of transmitted packets 647 required for successful Raptor decoding at the $m$ th 648 sink device.

Step 3) Calculate cost function $n_{p k t}^{c o r}\left(\vec{v}_{c n d}, c_{c n d}\right)$, and examine the 650 constraint in Eq. (13). $\phi_{i}^{\text {dec }}(s, k, c)$ can be calculated by 651

$$
\begin{aligned}
\phi_{i}^{\text {dec }}(s, k, c) & =P\left(X_{i}<n_{p k t}^{\min } \mid X \sim B\left(n_{i}^{r e c v}\left(v_{i}^{c n d}, c\right), 1-p l r_{i}\right)\right) \\
& =\sum_{i=0}^{n_{p k t}^{\min }-1}\left(\left(\begin{array}{c}
n_{i}^{\text {recv }}\left(v_{i}^{c n d}, c\right) \\
i
\end{array}\right)\left(1-p l r_{i}\right)^{i}\left(p l r_{i}\right)^{r_{i}^{r e c v}\left(v_{i}^{c n d}, c\right)-i}\right),
\end{aligned}
$$

$$
p l r_{i}=v_{i}^{\text {cnd }} \cdot p l r_{i}^{u n i}+\left(1-v_{i}^{\text {cnd }}\right) \cdot p l r_{i}^{\text {over }},
$$

where $X_{i}$ is the random variable of the number of 657 received packets at the $i$ th sink device, and $p l r_{i}$ is the 658 PLR at the $i$ th sink device [36], [37]. If the current 659 cost is smaller than the cost computed with the 660 parameters in $P_{c n d}^{t c}$, then the candidate parameters in 661 $P_{c n d}^{t c}$ are replaced with the current $\left(\vec{v}_{c n d}, c_{c n d}\right)$.

662 Step 4) Repeat Step 2 and 3 until all possible $m$ are examined. 663 Step 5) If all possible $j$ are examined, then terminate the process 664 with a solution in $P_{c n d}^{t c}$. Otherwise, go back to Step 1.

\subsubsection{Raptor Encoding Parameter Selection Algorithm 666}

We describe a method to determine the Raptor encoding 667 parameters (i.e., $n_{b l k}, s$, and $k$ ). In the Raptor encoding 668 parameter selection problem, the available set of $s$ and $k$ are 669 finite [37]. Moreover, $n_{b l k}$ can be determined by calculating 670 the maximum number of Raptor encoding blocks when $s 671$ and $k$ are given. Therefore, the solution that minimizes the 672 given cost function $e_{p k t}^{s e l}\left(n_{b l k}, s, k\right)$ can be obtained by con- 673 ducting a full search for all available set of $s$ and $k$. In fact, 674 the solution obtained by the proposed algorithm is a near 675 optimal solution for the optimal problem formulation, but it 676 is a feasible solution with a low computational complexity 677 for real-time processing. Details of the optimization proce- 678 dures are presented below, and the flow chart of the overall 679 parameter determining algorithm is shown in Fig. 4.

Step 0) Empty the candidate parameter set $P_{c n d}^{\text {rep }}$ and generate 681 the combinations of $(s, k)$.

Step 1) Select one of the generated combinations of $(s, k)$. 


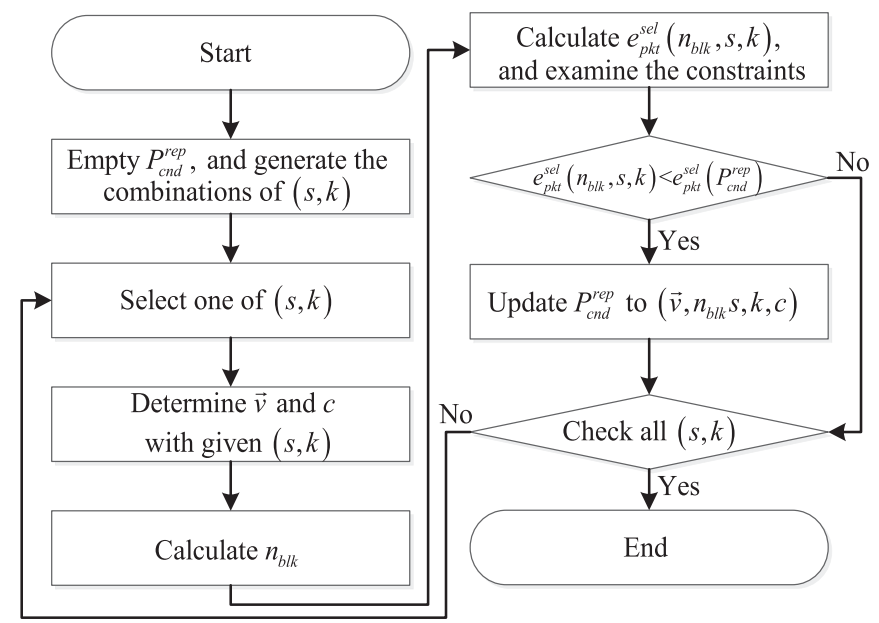

Fig. 4. Overall procedure of the parameter determining algorithm.
Step 2) Determine $\vec{v}$ and $c$ using the target sink device and code rate determining algorithm with the selected $(s, k)$.

Step 3) Calculate $n_{b l k}$ as follows.

$n_{b l k}= \begin{cases}\frac{\tilde{t}_{b u f}-t_{\text {ott }}-\max _{1 \leq i \leq N_{\text {sink }}} \tilde{t}_{i}^{\text {dec }}(s, k)}{\tilde{t}_{\text {enc }}(s, k, c)+t_{\text {blk }}} & \text { if } t_{b l k}^{\text {one }} \geq \max _{1 \leq i \leq N_{\text {sink }}} \tilde{t}_{i}^{\text {dec }}(s, k) \\ \frac{\tilde{t}_{\text {buf }}-t_{\text {ott }}-t_{\text {blk }}^{\text {one }}}{\tilde{t}_{\text {enc }}(s, k, c)+\max _{1 \leq i \leq N_{\text {sink }}} \tilde{t}_{i}^{\text {dec }}(s, k)} & \text { otherwise }\end{cases}$

$$
t_{b l k}^{o n e}=\frac{s \cdot k}{c \cdot r}
$$

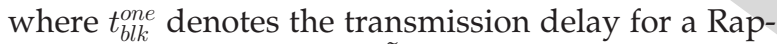
tor encoding block, and $\tilde{t}_{b u f}$ denotes the estimated buffered video playback time at the sink device. $\tilde{t}_{b u f}$ is calculate by

$$
\tilde{t}_{b u f}=\min _{1 \leq i \leq N_{\text {sink }}}\left(t_{i}^{b u f}+t_{i}^{o t t}\right)
$$

where $t_{i}^{\text {buf }}$ denotes the measured buffered video playback time at the $i$-th sink device, and $t_{i}^{\text {ott }}$ is the one-way trip time between the source device and the $i$ th sink device.

Step 4) Calculate the cost function $e_{p k t}^{s e l}\left(n_{b l k}, s, k\right)$, and examine the constraint in Eq. (14). If the current cost is smaller than the cost computed with the parameters in $P_{c n d}^{r e p}$, and the constraint is satisfied, then the candidate parameters in $P_{c n d}^{r e p}$ are replaced with the current parameters $\left(\vec{v}, n_{b l k}, s, k, c\right)$.

Step 5) If all possible combinations of $(s, k)$ are examined, then terminate the process with the optimal solution $P_{c n d}^{r e p}$. Otherwise, go back to Step 2.

\section{Experimental Results}

During the experiment, the proposed system is implemented using GStreamer [40] on a Linux-based single board computer, called ODROID [41], as shown in Fig. 5. The proposed system is examined over a real WiFi network with mobility. As illustrated in Fig. 6, we distributed one source device and four sink devices in our laboratory, and the source device moves around the laboratory during the experiment. For the real-time processing, two sink devices (Sink device \#1 and \#2) are running on ODROID-U3 equipped with a Samsung Exynos 4412 Prime Cortex-A9

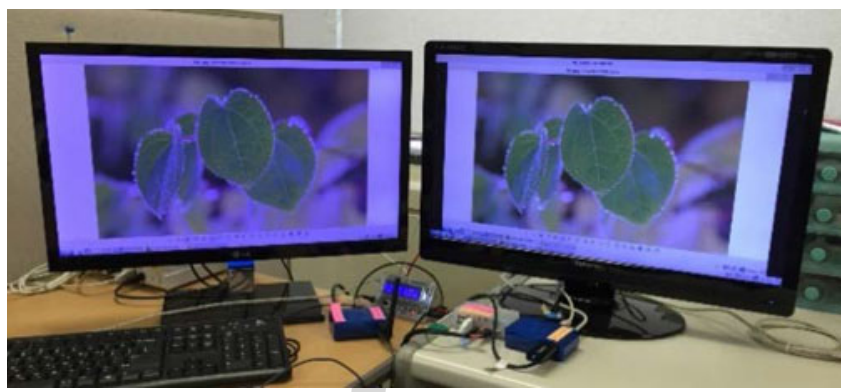

Fig. 5. Proposed system implemented on ODROID.

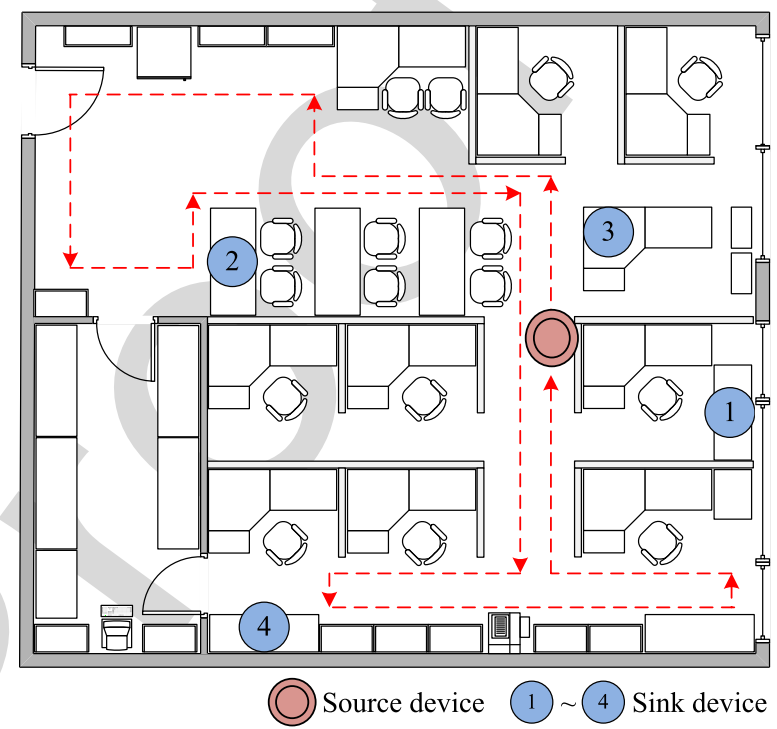

Fig. 6. Test environment of the proposed system (red dotted arrows indicate the moving route of the source device).

Quad Core 1.7 GHz processor and 2GB RAM. Other two 723 sink devices (Sink device \#3 and \#4) and a source device 724 are running on ODROID-XU4 equipped with a Samsung 725 Exynos 5422 Cortex-A15 Quad Core 2.0 GHz and Cor- 726 tex-A7 Quad Core 1.4 GHz processor with 2 GB RAM. 727 In the proposed system, Raptor codes are implemented 728 based on [25] with SIMD technology, and the traffic 729 shaping mechanism is implemented with reference to 730 Linux Traffic Control [48].

The experimental environment is set up as follows. For test 732 mirroring contents, we use a stored video, gallery application 733 that changes HD images every 5 seconds, and YouTube music 734 video [42]. The stored video is encoded by H.264 with an aver- 735 age of $5 \mathrm{Mbps}$ bit rate and 25 frames per second, and made by 736 combining the Pedestrian Area, Rush Hour, and Sunflower 737 videos [43]. The encoding structure is IPPPPPPPPPPP (i.e., 1738 GOP consists of 12 frames). The packet size $S_{p k t}$ is set to 739 1,024 bytes. The set of symbol sizes and set of number of sym- 740 bols are set to $\{64,128,256,512\}$ and $\{128,192,256,320,384,741$ $448,512\}$, respectively. To measure the consumed energy, an 742 ODROID Smart Power, which is a power measurement tool 743 for ODROID devices, is used [41]. Based on the power model 744 measurement methods in [37], the energy consumption 745 model parameters of the WiFi network interfaces are empiri- 746 cally measured by the ODROID Smart Power as presented in 747 Table 2. Figs. 7 and 8 show the measured data and models 748 obtained by using the curve fitting method. As shown in the 749 figures, their differences are very small. The model coeffi- 750 cients obtained by using the curve fitting methods are 751 
TABLE 2

Power Profiles of WiFi Network Interfaces

\begin{tabular}{lcccc}
\hline \multicolumn{2}{c}{ Source device } & & \multicolumn{2}{c}{ Sink device } \\
\cline { 5 - 6 } Parameter & Value & & Parameter & Value \\
\hline$\beta_{\text {src }}, \gamma_{\text {src }}$ & $2.90,396.97$ & & $\beta_{i}^{\text {sink }}, \gamma^{\text {sink }}$ & $2.67,761.02$ \\
$p_{\text {sea }}^{\text {bar }}$ & $370 \mathrm{~mW}$ & & $p_{i}^{\text {tail }}$ & $720.56 \mathrm{~mW}$ \\
$T_{\text {bea }}$ & $100 \mathrm{~ms}$ & & $p_{i}^{\text {chn }}$ & $420.54 \mathrm{~mW}$ \\
$T_{\text {intv }}$ & $100 \mathrm{~ms}$ & & $T_{i}^{\text {tail }}$ & $150 \mathrm{~ms}$ \\
\hline
\end{tabular}

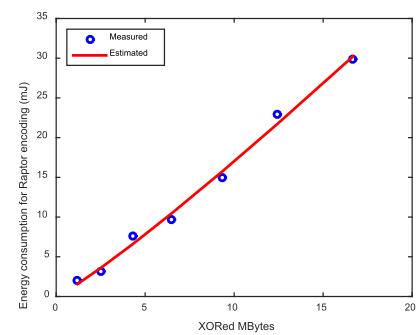

(a)

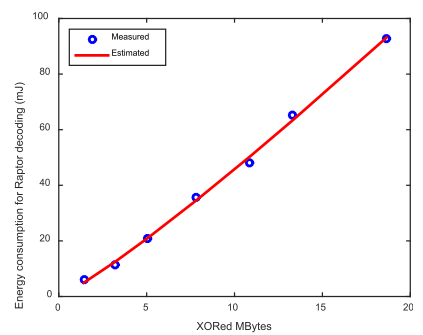

(b)
Fig. 7. Examples of the curve fitting for the energy consumption model of Raptor encoding/decoding when $\mathrm{s}=128$ : (a) Energy consumption of Raptor encoding at source device. (b) Energy consumption of Raptor decoding at sink device \#1.

TABLE 3

Coefficients of Energy Consumption and Delay Model for Raptor Encoding and Decoding

\begin{tabular}{llllllll}
\hline$s$ & \multicolumn{3}{l}{ Encoding (Source device) } & & \multicolumn{3}{c}{ Decoding (Sink device \#1) } \\
\cline { 2 - 3 } \cline { 6 - 7 } & $\rho_{\text {enc }}(s)$ & $\mu_{\text {enc }}(s)$ & $\sigma_{\text {enc }}(s)$ & & $\rho_{i}^{\text {dec }}(s)$ & $\mu_{i}^{\text {dec }}(s)$ & $\sigma_{i}^{\text {dec }}(s)$ \\
\hline 64 & 0.00149 & 1.1560 & 0.6235 & & 0.00447 & 1.3070 & 2.7300 \\
128 & 0.00128 & 1.1230 & 0.4555 & & 0.00331 & 1.1410 & 1.4486 \\
256 & 0.00136 & 1.0210 & 0.3885 & & 0.00170 & 1.1330 & 0.7950 \\
512 & 0.00170 & 0.9302 & 0.3269 & & 0.00157 & 1.0160 & 0.4855 \\
\hline
\end{tabular}

presented in Table 3. These parameters are embedded in the implemented system.

For the performance comparison, the peak signal-tonoise ratio (PSNR) between the original screen content and the received screen content is adopted as an objective spatial video quality metric. The average PSNR denotes the average of PSNRs observed at four sink devices. Furthermore, the control packet overhead for feedback information is adopted as a system overhead metric. The control overhead $P O_{c t r l}$ is defined as follows:

$$
P O_{c t r l}=\frac{n_{b y t e}^{c t r l}}{n_{p k t}^{\text {data }} \cdot S_{p k t}+n_{\text {byte }}^{c t r l}},
$$

where $n_{p k t}^{d a t a}$ is the number of transmitted data packets including video data packets, redundant packets, and retransmitted packet, and $n_{\text {byte }}^{c t r l}$ is the total size of the transmitted control packets from the source device. The control packets include all packets except for data packets.

\subsection{Energy Consumption and Delay Model Verification}

In this section, we present the model verification of the energy consumption and delay models. During the experiment, stored video is used as the test mirroring content. The experimental results are captured at the source device and sink device \#1. First, we examine how well the energy

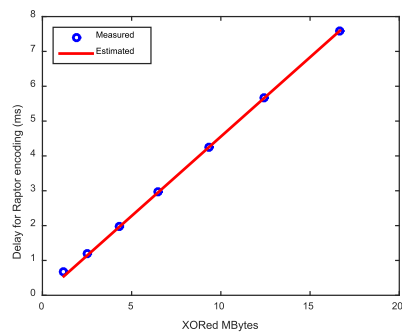

(a)

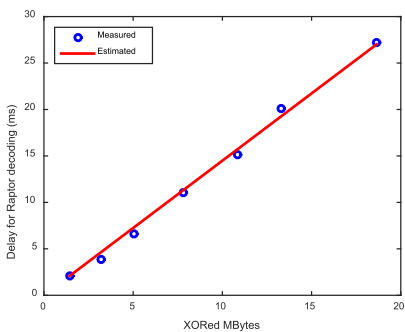

(b)
Fig. 8. Examples of the curve fitting for the delay model of Raptor encoding/decoding when $s=128$ : (a) Delay of Raptor encoding at source device. (b) Delay of Raptor decoding at sink device \#1.

TABLE 4

Measured and Estimated Energy Consumption According to the Amount of Transmitted Data in a Bin

\begin{tabular}{|c|c|c|c|c|}
\hline \multirow{2}{*}{$\begin{array}{l}\text { Amount of } \\
\text { transmitted } \\
\text { data (Mbits) }\end{array}$} & \multicolumn{2}{|c|}{ Source device } & \multicolumn{2}{|c|}{ Sink device \#1 } \\
\hline & $\begin{array}{c}\text { Measured } \\
\text { energy }(\mathrm{mJ})\end{array}$ & $\begin{array}{c}\text { Estimated } \\
\text { energy }(\mathrm{mJ})\end{array}$ & $\begin{array}{c}\text { Measured } \\
\text { energy }(\mathrm{mJ})\end{array}$ & $\begin{array}{c}\text { Estimated } \\
\text { energy }(\mathrm{mJ})\end{array}$ \\
\hline 5 & 3130.50 & 3011.96 & 5979.45 & 5491.83 \\
\hline 10 & 3580.77 & 3502.33 & 6725.18 & 6947.69 \\
\hline 15 & 3938.93 & 3844.42 & 7324.13 & 7219.24 \\
\hline 20 & 4276.66 & 4431.41 & 8144.21 & 7609.94 \\
\hline 25 & 4799.70 & 4879.67 & 8664.43 & 8380.34 \\
\hline 30 & 5342.65 & 5018.58 & 9150.55 & 9377.65 \\
\hline
\end{tabular}

consumption model of the WiFi network interface fits the 776 observed experimental data. During the experiment, the 777 bin duration and the transmission rate are set to 10 seconds 778 and $5 \mathrm{Mbps}$, respectively. Table 4 presents the comparison 779 between the measured and estimated energy consumption in 780 terms of the amount of transmitted data in a bin. It is appar- 781 ently observed that the estimated energy fits well with the 782 measured energy. However, an estimation error still exists 783 between the measured energy and estimated energy, due to 784 inaccurate estimation of network conditions and processing 785 power consumption. The average estimation error rates (i.e., 786 |estimated value - measured value $\mid \times 100 /$ estimated value) at 787 the source device and sink device are approximately 3.29788 and 4.20 percent, respectively.

Now, we investigate the accuracy of the SIMD-based 790 energy consumption and delay models for Raptor encoding 791 and decoding processes. Table 5 shows the measured and 792 estimated energy consumption of Raptor encoding and 793 decoding processes according to the symbol size and the 794 number of source symbols. The amount of XORed bytes is 795 obtained by multiplying the symbol size by the total num- 796 ber of symbol-level XOR operations. It is obviously shown 797 that the energy consumption of Raptor encoding and 798 decoding processes increases as the amount of XORed 799 bytes increases. The average estimation error rates of the 800 energy consumption of the Raptor encoding and decoding 801 processes are approximately 9.07 and 8.12 percent, res- 802 pectively. Table 6 presents the measured and estimated 803 Raptor encoding and decoding delays. The Raptor encod- 804 ing and decoding delay linearly increase as the amount 805 of XORed bytes increases. The average estimation error 806 rates of the Raptor encoding and decoding delay are 807 approximately 6.39 and 5.54 percent, respectively. Conse- 808 quently, we can say that the Raptor codes energy con- 809 sumption model and delay models have a good fit with 810 the observed data. 
TABLE 5

Measured and Estimated Raptor Encoding/Decoding Energy According to Symbol Parameters

\begin{tabular}{|c|c|c|c|c|c|}
\hline \multirow[t]{2}{*}{$s$} & \multirow[t]{2}{*}{$k$} & \multicolumn{2}{|c|}{ Encoding (Source device) } & \multicolumn{2}{|c|}{ Decoding (Sink device \#1) } \\
\hline & & $\begin{array}{c}\text { Measured } \\
\text { energy }(\mathrm{mJ})\end{array}$ & $\begin{array}{c}\text { Estimated } \\
\text { energy }(\mathrm{m} J)\end{array}$ & $\begin{array}{c}\text { Measured } \\
\text { energy }(\mathrm{mJ})\end{array}$ & $\begin{array}{c}\text { Estimated } \\
\text { energy }(\mathrm{mJ})\end{array}$ \\
\hline \multirow[t]{7}{*}{128} & 128 & 2.02 & 1.57 & 6.17 & 4.97 \\
\hline & 192 & 3.14 & 3.64 & 11.41 & 12.56 \\
\hline & 256 & 7.60 & 6.62 & 20.73 & 21.06 \\
\hline & 320 & 9.69 & 10.48 & 35.76 & 34.45 \\
\hline & 384 & 14.98 & 15.72 & 48.07 & 50.13 \\
\hline & 448 & 22.89 & 21.73 & 65.09 & 63.42 \\
\hline & 512 & 29.88 & 30.23 & 92.80 & 93.15 \\
\hline \multirow[t]{7}{*}{256} & 128 & 3.05 & 3.31 & 6.60 & 5.58 \\
\hline & 192 & 6.25 & 7.12 & 14.45 & 13.99 \\
\hline & 256 & 14.41 & 12.26 & 22.47 & 23.37 \\
\hline & 320 & 19.07 & 18.60 & 37.13 & 38.10 \\
\hline & 384 & 24.72 & 26.89 & 54.06 & 55.29 \\
\hline & 448 & 36.47 & 36.08 & 72.81 & 69.82 \\
\hline & 512 & 49.03 & 48.70 & 101.34 & 102.27 \\
\hline \multirow[t]{7}{*}{512} & 128 & 9.13 & 7.31 & 15.57 & 9.23 \\
\hline & 192 & 14.42 & 14.70 & 20.68 & 21.06 \\
\hline & 256 & 29.07 & 24.13 & 33.93 & 33.37 \\
\hline & 320 & 33.98 & 35.27 & 53.98 & 51.73 \\
\hline & 384 & 44.39 & 49.35 & 64.89 & 72.26 \\
\hline & 448 & 64.23 & 64.52 & 89.94 & 89.09 \\
\hline & 512 & 86.94 & 84.80 & 127.64 & 125.48 \\
\hline
\end{tabular}

TABLE 6

Measured and Estimated Raptor Encoding/Decoding Delay According to Symbol Parameters

\begin{tabular}{|c|c|c|c|c|c|}
\hline \multirow[t]{2}{*}{$s$} & \multirow{2}{*}{$k$} & \multicolumn{2}{|c|}{ Encoding (Source device) } & \multicolumn{2}{|c|}{ Decoding (Sink device \#1) } \\
\hline & & $\begin{array}{l}\text { Measured } \\
\text { delay (ms) }\end{array}$ & $\begin{array}{l}\text { Estimated } \\
\text { delay (ms) }\end{array}$ & $\begin{array}{l}\text { Measured } \\
\text { delay }(\mathrm{ms})\end{array}$ & $\begin{array}{r}\text { Estimated } \\
\text { delay (ms) }\end{array}$ \\
\hline \multirow[t]{7}{*}{128} & 128 & 0.67 & 0.55 & 2.13 & 2.07 \\
\hline & 192 & 1.19 & 1.15 & 3.82 & 4.67 \\
\hline & 256 & 1.97 & 1.97 & 6.63 & 7.34 \\
\hline & 320 & 2.97 & 2.96 & 11.08 & 11.30 \\
\hline & 384 & 4.24 & 4.25 & 15.13 & 15.70 \\
\hline & 448 & 5.67 & 5.66 & 20.07 & 19.29 \\
\hline & 512 & 7.57 & 7.60 & 27.21 & 27.02 \\
\hline \multirow[t]{7}{*}{256} & 128 & 1.11 & 0.93 & 2.54 & 2.27 \\
\hline & 192 & 2.20 & 1.97 & 4.55 & 5.12 \\
\hline & 256 & 3.58 & 3.35 & 7.48 & 8.06 \\
\hline & 320 & 5.28 & 5.05 & 11.97 & 12.40 \\
\hline & 384 & 7.38 & 7.24 & 17.05 & 17.23 \\
\hline & 448 & 9.65 & 9.66 & 22.32 & 21.17 \\
\hline & 512 & 12.69 & 12.96 & 29.36 & 29.66 \\
\hline \multirow[t]{7}{*}{512} & 128 & 1.99 & 1.56 & 3.21 & 2.78 \\
\hline & 192 & 3.82 & 3.31 & 5.78 & 6.25 \\
\hline & 256 & 6.14 & 5.65 & 9.26 & 9.84 \\
\hline & 320 & 8.93 & 8.49 & 15.18 & 15.15 \\
\hline & 384 & 12.40 & 12.19 & 20.55 & 21.05 \\
\hline & 448 & 16.23 & 16.25 & 26.58 & 25.86 \\
\hline & 512 & 21.30 & 21.80 & 36.19 & 36.23 \\
\hline
\end{tabular}

\subsection{Performance Verification According to Various Parameters}

The performance of the proposed system according to various parameters is provided. During the experiment, the source device and all sink devices remain stationary in the laboratory. $T_{\text {play }}^{\max }$ is set to 500,1000 , and $1500 \mathrm{~ms}$ for the performance comparison according to $T_{\text {play }}^{\max }$. The stored video is
TABLE 7

Performance Comparison with Fixed Raptor Encoding Parameters

\begin{tabular}{lccccccc}
\hline $\begin{array}{l}\text { Param. } \\
\text { setting }\end{array}$ & $n_{\text {blk }}$ & $s$ & $k$ & $\begin{array}{c}T_{\text {play }}^{\max } \\
(\mathrm{ms})\end{array}$ & $\begin{array}{c}\text { Total } \\
\text { energy (J) }\end{array}$ & $\begin{array}{c}\text { Avg. } \\
\text { PSNR (dB) }\end{array}$ & $\begin{array}{c}\text { Playback } \\
\text { delay (ms) }\end{array}$ \\
\hline $\begin{array}{l}\text { Fixed } \\
\text { param. }\end{array}$ & 32 & 64 & 128 & - & 2387.56 & 43.50 & 332 \\
& 8 & 128 & 256 & - & 2407.01 & 43.50 & 375 \\
& 2 & 256 & 512 & - & 2657.46 & 43.50 & 384 \\
& 64 & 64 & 128 & - & 2328.97 & 43.50 & 631 \\
& 16 & 128 & 256 & - & 2345.26 & 43.50 & 738 \\
& 4 & 256 & 512 & - & 2589.82 & 43.50 & 754 \\
& 128 & 64 & 128 & - & 2309.35 & 43.50 & 1283 \\
& 32 & 128 & 256 & - & 2314.23 & 43.50 & 1481 \\
Adaptive & - & 256 & 512 & - & 2495.90 & 43.50 & 1488 \\
param. & & - & - & 500 & 2282.13 & 43.50 & 415 \\
& - & - & - & 1000 & 2230.32 & 43.50 & 974 \\
& - & - & - & 1500 & 2192.43 & 43.50 & 1463 \\
\hline
\end{tabular}

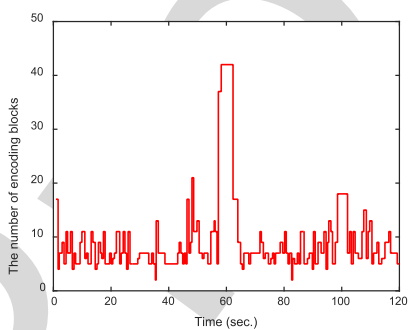

(a)

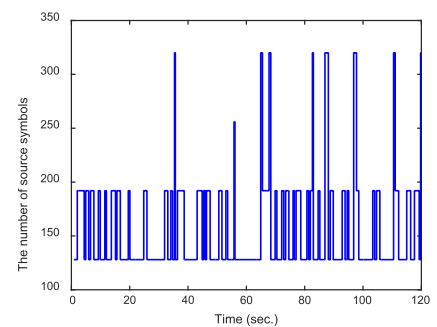

(c)
Fig. 9. Raptor encoding parameters of the proposed system: (a) Number of Raptor encoding blocks, (b) symbol size, and (c) number of source symbols (captured at source device).

used as the test mirroring content. First, we describe the 819 performance comparison conducted with the fixed Raptor 820 encoding parameters in Table 7. It is clearly shown that the 821 total energy consumption of the source device and four sink 822 devices decreases as the block size (i.e., $n_{b l k} \cdot s \cdot k$ ) increases. 823 This is because the WiFi network interface can spend more 824 time in the inactive state by traffic shaping. However, the 825 playback delay increases proportionally to the block size 826 because more time is required to transmit the block and 827 perform Raptor encoding and decoding processes. Based 828 on this phenomenon, the proposed system dynamically 829 adjusts the number of Raptor encoding blocks, the symbol 830 size, and the number of source symbols by considering the 831 network conditions, as shown in Fig. 9 ( $T_{\text {plax }}^{\max }$ is $500 \mathrm{~ms}$ ). As 832 shown in the figure, the proposed system selects $s$ and $k 833$ from the given set of the symbol sizes and set of number of 834 symbols. The number of Raptor encoding blocks is adap- 835 tively determined according to the estimated network con- 836 ditions and buffered video playback time. Moreover, as 837 


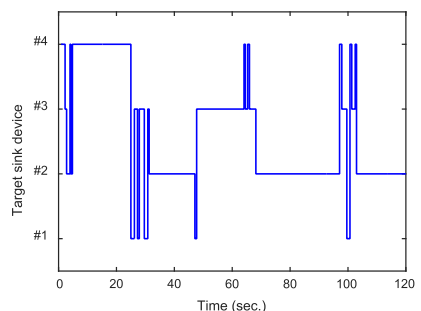

(a)

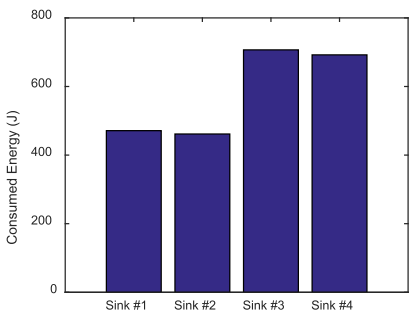

(c)

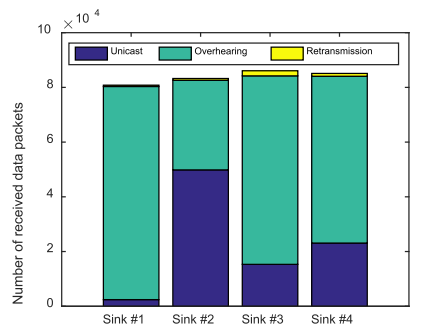

(b)

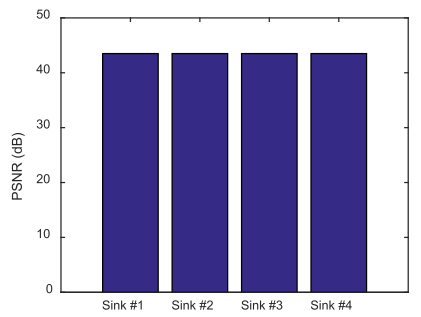

(d)

Fig. 10. Performance comparison according to sink device when the source device moves: (a) selected target sink device, (b) number of received data packets, (c) consumed energy, and (d) PSNR.

shown in Table 7 , the playback delay between the source device and sink device can be controlled by $T_{\text {play }}^{\max }$ (i.e., determined by user preference) in the proposed system. As $T_{\text {play }}^{\max }$ increases, the playback delay increases while the energy consumption is reduced, and vice versa. Thus, the proposed system can achieve good energy efficiency while supporting a high-quality screen mirroring service.

We investigate the performance comparison of the proposed system according to the sink device when the source device is moving around. $T_{\text {play }}^{\max }$ is set to $500 \mathrm{~ms}$, and the stored video is used as the test mirroring content. As shown in Fig. 10a, sink device \#2 is selected as the target sink device more often than the others, so sink device \#2 receives most of the data packets using unicast as shown in Fig. 10b. Mean-

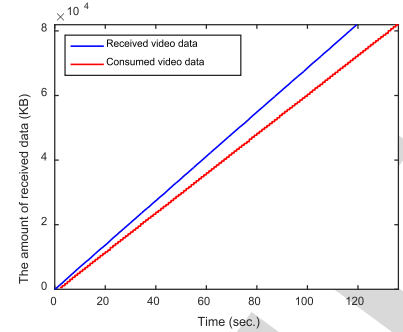

(a)

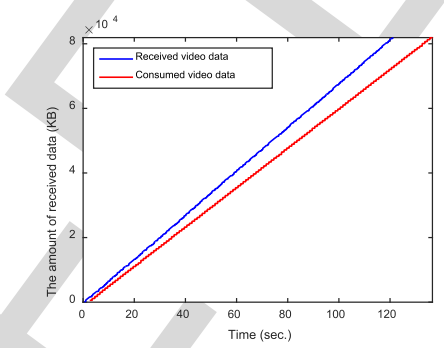

(b)

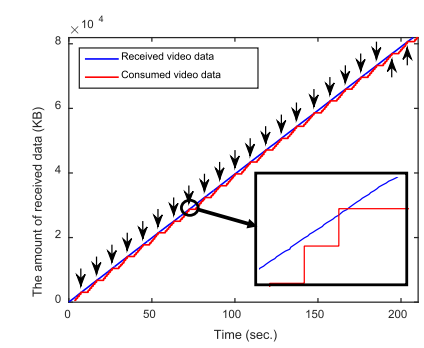

(c)

while, sink device \#1 receives most of the data packets using 852 overhearing because the time selected as the target sink 853 device is the shortest. However, since the PLR of overhearing 854 at sink device \#1 is very low, most of the data packets can be 855 received without redundant packets and retransmission 856 packets. On the other hand, sink device \#3 requires more 857 redundant packets and retransmission packets than sink 858 device \#1 and \#2 in order to recover lost packets. Due to this, 859 it consumes more energy than that of sink device \#1 and \#2, 860 as shown in Fig. 10c. In particular, sink device \#3 requires the 861 largest amount of data and energy because its PLR is the low- 862 est among the sink devices. All sink devices can successfully 863 recover the lost packets and provide high quality screen mir- 864 roring service as shown in Fig. 10d.

\subsection{Performance Comparison with Existing Systems}

The performance of the proposed system is compared with 868 that of three existing systems, namely Pseudo-broadcast [29], 869 DirCast [12], and ACK-based multicast [44], which are modi- 870 fied slightly for our experiment. For a fair comparison, DirCast 871 employs Raptor codes instead of the Reed Solomon codes [45], 872 which is originally adopted in DirCast. During the experi- 873 ment, the source device continuously moves around the labo- 874 ratory, and all sink devices remain stationary, as shown in 875 Fig. 6. For the experiment, we set $T_{\text {play }}^{\max }$ to $500 \mathrm{~ms}$ based on [2]. 876 First, we examine the cumulative curves of the received video 877 data and consumed video data. During the experiment, the 878 test mirroring contents are stored video. The results are pre- 879 sented in Fig. 11, and the arrows in the figure indicate the 880 period of buffer underflow. As shown in the figure, it is obvi- 881 ously shown that the proposed system, Pseudo-broadcast, 882 and DirCast can provide seamless video streaming without 883 buffer underflow, whereas ACK-based multicast experience 884 buffer underflows that result in frozen video.

The PSNR comparisons with existing protocols are pre- 886 sented in Fig. 12. It is clearly indicated in the figure that the 887

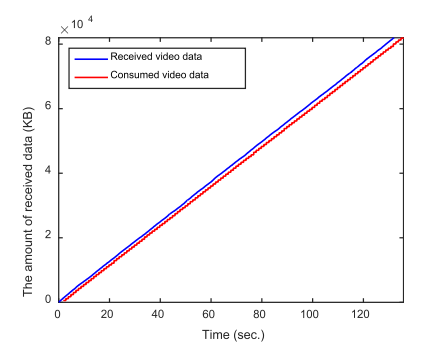

(d)

Fig. 11. Cumulative curves of received video data and consumed video data (captured at sink device \#4): (a) Pseudo-broadcast, (b) DirCast, (c) ACK-based multicast, and (d) proposed system.

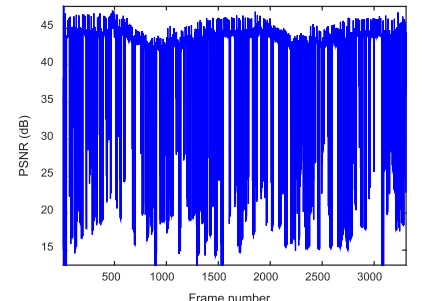

(a)

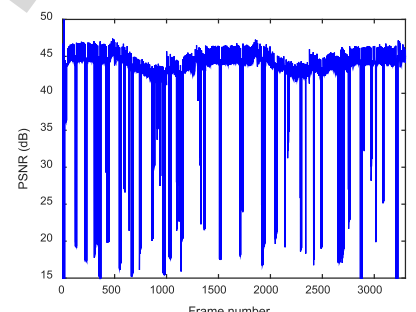

(b)

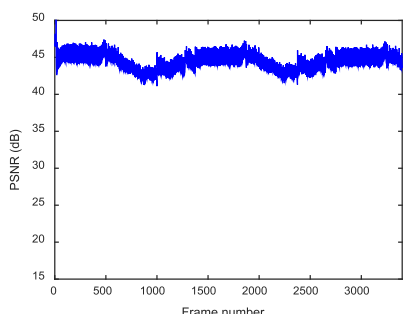

(c)

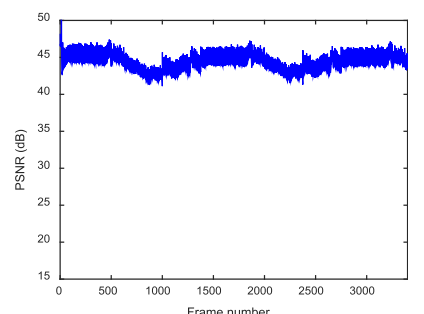

(d)

Fig. 12. PSNR comparison with existing systems (captured at sink device \#4): (a) Pseudo-broadcast, (b) DirCast, (c) ACK-based multicast, and (d) proposed system. 


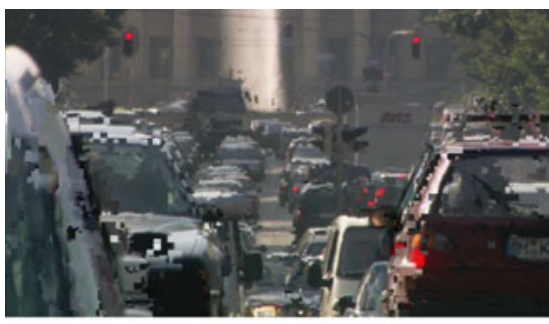

(a)

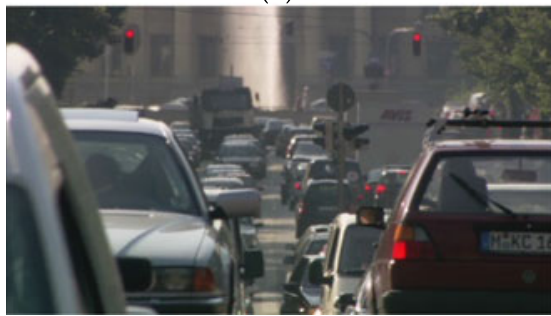

(c)

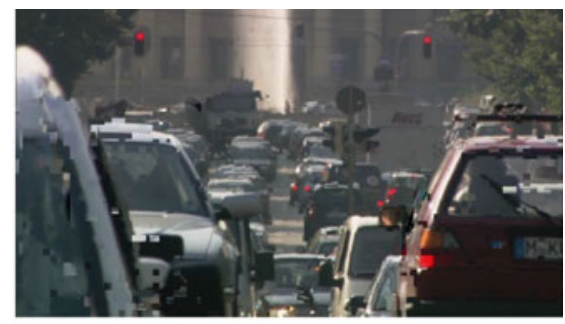

(b)

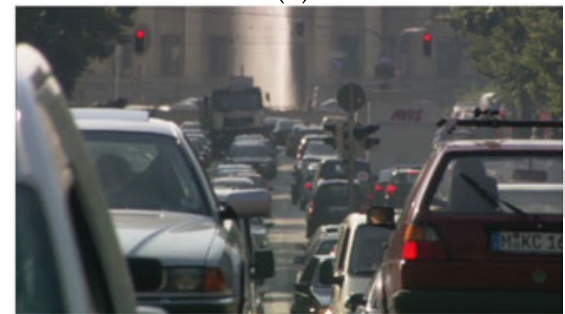

(d)

Fig. 13. Subjective video quality comparison of 1362nd frame (captured at sink device \#4): (a) Pseudo-broadcast, (b) DirCast, (c) ACK-based multicast, and (d) proposed system.

TABLE 8

Energy and PSNR Results Per Device

\begin{tabular}{|c|c|c|c|c|c|c|c|c|c|c|}
\hline \multirow[t]{2}{*}{ Mirroring Contents } & \multirow[t]{2}{*}{ System } & \multicolumn{5}{|c|}{ Energy $(\mathrm{J})$} & \multicolumn{4}{|c|}{ PSNR (dB) } \\
\hline & & Source & Sink \#1 & Sink \#2 & Sink \#3 & Sink \#4 & Sink \#1 & Sink \#2 & Sink \#3 & Sink \#4 \\
\hline \multirow[t]{4}{*}{ Stored video } & Pseudo-broadcast & 568.37 & 359.59 & 349.12 & 539.39 & 523.68 & 21.43 & 35.85 & 35.85 & 30.74 \\
\hline & DirCast & 709.37 & 468.47 & 462.46 & 702.70 & 693.68 & 41.81 & 40.70 & 41.32 & 42.39 \\
\hline & ACK-based multicast & 1139.66 & 692.43 & 676.86 & 1038.64 & 1015.29 & 43.50 & 43.50 & 43.50 & 43.50 \\
\hline & Proposed system & 706.45 & 471.20 & 461.51 & 706.80 & 692.26 & 43.50 & 43.50 & 43.50 & 43.50 \\
\hline \multirow[t]{4}{*}{ Gallery application } & Pseudo-broadcast & 924.71 & 377.32 & 377.43 & 565.98 & 566.15 & 29.34 & 29.34 & 17.76 & 29.42 \\
\hline & DirCast & 1289.88 & 513.10 & 527.34 & 769.66 & 791.01 & 33.83 & 41.16 & 41.27 & 36.13 \\
\hline & ACK-based multicast & 1941.41 & 784.90 & 786.15 & 1177.34 & 1179.23 & 41.57 & 41.57 & 41.57 & 41.57 \\
\hline & Proposed system & 1120.53 & 455.65 & 456.57 & 683.48 & 684.85 & 41.57 & 41.57 & 41.57 & 41.57 \\
\hline \multirow[t]{4}{*}{ YouTube music video } & Pseudo-broadcast & 1032.99 & 424.07 & 436.14 & 636.11 & 654.20 & 32.56 & 29.90 & 29.90 & 19.30 \\
\hline & DirCast & 1325.84 & 550.92 & 549.13 & 826.39 & 823.69 & 33.42 & 32.47 & 35.14 & 34.02 \\
\hline & ACK-based multicast & 2073.18 & 811.87 & 802.00 & 1217.80 & 1203.00 & 41.44 & 41.44 & 41.44 & 41.44 \\
\hline & Proposed system & 1160.93 & 478.89 & 481.51 & 718.34 & 722.27 & 41.44 & 41.44 & 41.44 & 41.44 \\
\hline
\end{tabular}

TABLE 9

Summary of Performance Comparison with Existing Systems

\begin{tabular}{|c|c|c|c|c|c|c|}
\hline Mirroring Contents & System & $\begin{array}{c}\text { Total } \\
\text { Energy }(\mathrm{J})\end{array}$ & $\begin{array}{c}\text { Avg. } \\
\text { PSNR (dB) }\end{array}$ & $\begin{array}{l}\text { Std. } \\
\text { PSNR }\end{array}$ & $\begin{array}{c}\text { Avg. Number } \\
\text { of buffer } \\
\text { underflows }\end{array}$ & $\begin{array}{l}\text { Avg. Control } \\
\text { overhead }(\%)\end{array}$ \\
\hline \multirow[t]{4}{*}{ Stored video } & Pseudo-broadcast & 2340.15 & 30.97 & 6.80 & 0 & 0.45 \\
\hline & DirCast & 3036.68 & 41.56 & 0.72 & 0 & 0.55 \\
\hline & ACK-based multicast & 4562.88 & 43.50 & 0 & 22 & 6.26 \\
\hline & Proposed system & 3038.22 & 43.50 & 0 & 0 & 2.06 \\
\hline \multirow[t]{4}{*}{ Gallery application } & Pseudo-broadcast & 2811.60 & 26.47 & 5.80 & 0 & 0.45 \\
\hline & DirCast & 3891.00 & 38.10 & 3.72 & 0 & 0.53 \\
\hline & ACK-based multicast & 5869.03 & 41.57 & 0 & 20 & 6.27 \\
\hline & Proposed system & 3401.09 & 41.57 & 0 & 0 & 1.38 \\
\hline \multirow[t]{4}{*}{ YouTube music video } & Pseudo-broadcast & 3183.50 & 27.92 & 5.88 & 0 & 0.53 \\
\hline & DirCast & 4075.97 & 33.76 & 1.12 & 0 & 0.49 \\
\hline & ACK-based multicast & 6107.85 & 41.44 & 0 & 21 & 6.25 \\
\hline & Proposed system & 3561.94 & 41.44 & 0 & 0 & 0.97 \\
\hline
\end{tabular}

video quality of Pseudo-broadcast is frequently and seriously degraded because Pseudo-broadcast does not support an error correction method. Although DirCast supports an error correction method, it is observed that the video quality is somewhat degraded because DirCast cannot finely adjust code rates considering the time-varying wireless network 893 environment. Furthermore, DirCast cannot completely 894 recover lost packets when unexpected extreme losses occur. 895 Conversely, the proposed system and ACK-based multicast 896 recover most lost packets successfully and support the 897 
screen mirroring service without any noticeable video quality degradation. For a subjective video quality comparison, the captured 1,362nd frame of the stored video are presented in Fig. 13. It is obviously shown that the video quality of both the proposed system and ACK-based multicast is much better than that of either Pseudo-broadcast or DirCast.

The energy and PSNR results per device and the summary of performance comparison with existing systems are shown in Tables 8 and 9, respectively. As shown in Tables 8 and 9, the proposed system and ACK-based multicast can support high-quality screen mirroring services with a $4.37 \mathrm{~dB}$ improvement on average compared to DirCast. The PSNR of the proposed system is 32.59 percent higher than Pseudo-broadcast and 10.45 percent higher than DirCast. Moreover, the proposed system and ACK-based multicast provide equal level of PSNR quality for all sink devices because they utilize lost packet recovery schemes. In the ACK-based multicast, the source device repeatedly transmits the lost video data until all sink devices transmit an ACK message that notifies the source device of successful reception. Thus, ACK-based multicast can provide reliable screen mirroring service without video quality degradation; however, it requires more time to receive the video data because the transmission of the next video data can be delayed at the source device until the source device receives ACK messages for the current video data from all sink devices. During the experiment, underflow occurs 21 times on average for ACK-based multicast. Meanwhile, in the case of the proposed system, most lost packets are recovered by the Raptor codes with no delay of retransmission requests and a small number of unexpected lost packets are recovered by the NACK-based retransmission request scheme with minimal delays. Thus, the proposed system cannot only provide reliable screen mirroring service, but can also provide seamless screen mirroring services without buffer underflow. Moreover, the proposed system requires a reasonable amount of control overhead compared to existing systems. As shown in Table 9, the control overhead of the proposed system is average four times lower than ACKbased multicast. As shown in Tables 8 and 9, the proposed system consumes less energy than the FEC-based systems, i.e., DirCast and ACK-based multicast. The proposed system can save 8.38 percent of energy consumption compared to DirCast. In addition, it is shown that the proposed system can provide an energy saving of 39.05 percent compared to ACK-based multicast while providing a similar level of video quality. Consequently, the proposed system can provide a reliable and energy-efficient hybrid screen mirroring service with relatively low control overhead.

\section{Conclusion}

In this paper, we have proposed a reliable and energyefficient hybrid screen mirroring multicast system for sharing high-quality screen mirroring service among adjacent sink devices. In the proposed system, systematic Raptor codes and NACK-based retransmission are employed to reduce the video quality degradation over an error-prone WiFi network. The proposed system not only shapes the screen mirroring traffic, but also determines the target sink device and Raptor encoding parameters while considering the energy consumption of the source device and sink devices. The proposed system has been fully implemented in Linux-based single board computers, and tested over a real WiFi network. Experimental results show that the pro- 960 posed system can provide energy savings of 39.05 percent 961 compared to ACK-based multicast systems while provid- 962 ing the same level of video quality. Furthermore, the pro- 963 posed system can provide high-quality screen mirroring 964 without noticeable video quality degradation compared to 965 existing systems.

\section{ACKNOWLEDGMENTS}

This research was supported by the Basic Science Research 968 Program through the National Research Foundation of 969 Korea(NRF) funded by the Ministry of Science, ICT \& 970 Future Planning(NRF-2016R1A2B4007812).

\section{References}

[1] Screen mirroring awareness reaches 40 percent of smartphone 973 and tablet owners. [Online]. Available: https://www.npd.com/ 974 $\mathrm{wps} / \mathrm{portal} / \mathrm{npd} / \mathrm{us} / \mathrm{news} /$ press-releases/screen-mirroring- 975 awareness-reaches-40-percent-of-smartphone-and-tablet-owners- 976 according-to-the-npd-group/, Accessed on: 2017.

[2] C. Hsu, T. Tsai, C. Huang, C. Hsu, and K. Chen, "Screencast dis- 978 sected: Performance measurements and design considerations," 979 in Proc. ACM Conf. Multimedia Syst., Mar. 2015, pp. 177-188. 980

[3] C. F. Lai, H. Wang, H.-C. Chao, and G. Nan, "A network and 981 device aware QoS approach for cloud-based mobile streaming," 982 IEEE Trans. Multimedia, vol. 15, no. 4, pp.747-757, Jun. 2013.

[4] S. Wang and S. Dey, "Adaptive mobile cloud computing to enable 984 rich mobile multimedia applications," IEEE Trans. Multimedia, 985 vol. 15, no. 4, pp. 870-883, Jun. 2013.

[5] C.-Y. Huang, K.-T. Chen, D.-Y. Chen, H.-J. Hsu, and C.-H. Hsu, 987 "GamingAnywhere: The first open source cloud gaming system," 988 ACM Trans. Multimedia Comput., vol. 10, no. 1, pp. 173-185, Jan. 989 2014.

[6] Use AirPlay or AirPlay Mirroring on your iPhone, iPad, or iPod 991 touch. [Online]. Available: https://support.apple.com/en-us / 992 HT204289/, Accessed on: 2017.

[7] Chromecast. [Online]. Available: https://www.google.com/intl/ 994 en_us/chromecast/?utm_source $=$ chromecast.com/, Accessed on: 995 2017.

[8] MirrorOp. [Online]. Available: http://www.mirrorop.com/, 997 Accessed on: 2017.

[9] Splashtop. [Online]. Available: http://www.splashtop.com/, 999 Accessed on: 2017.

[10] Wi-Fi Certified Miracast. [Online]. Available: https://www.wi-fi. 1001 org/discover-wi-fi/wi-fi-certified-miracast/, Accessed on: 2017. 1002

[11] S. Sen, N. K. Madabhushi, and S. Banerjee, "Scalable WiFi media 1003 delivery through adaptive broadcasts," in Proc. USENIX Symp. 1004 Netw. Syst. Des. Implementation, Apr. 2010, pp. 191-204.

[12] R. Chandra, et al., "DirCast: A practical and efficient Wi-Fi multi- 1006 cast system," in Proc. IEEE Int. Conf. Netw. Protocols, Oct. 2009, 1007 pp. 161-170.

[13] J. C. MacKay, "Fountain codes," IEEE Proc. Commun., vol. 152, 1009 no. 6, pp. 1062-1068, Dec. 2005.

[14] S. Ahmad, R. Hamzaoui, and M. M. Al-Akaidi, "Unequal error 1011 protection using fountain codes with applications to video 1012 communication," IEEE Trans. Multimedia, vol. 13, no. 1, pp. 92- 1013 101, Feb. 2011.

[15] Z. Luo, L. Song, S. Zheng, and N. Ling, "Raptor codes based 1015 unequal protection for compressed video according to packet pri- 1016 ority," IEEE Trans. Multimedia, vol. 15, no. 8, pp. 2208-2213, 1017 Dec. 2013.

[16] C.-H. Lin, C.-K. Shieh, and W.-S. Hwang, "An access point- 1019 based FEC mechanism for video transmission over wireless 1020 LANs," IEEE Trans. Multimedia, vol. 15, no. 1, pp. 195-206, 1021 Jan. 2013.

[17] A. Hameed, R. Dai, and B. Balas, "A decision-tree-based percep- 1023 tual video quality prediction model and its application in FEC for 1024 wireless multimedia communications," IEEE Trans. Multimedia, 1025 vol. 18, no. 4, pp. 764-774, Apr. 2016.

[18] Why Digital Fountain's Raptor Code Is Better Than Reed Solomon 1027 Erasure Codes For Streaming Applications, Digital Fountain, Inc., 1028 Fremont, CA, USA, 2005. 
[19] M. A. Hoque, M. Siekkinen, and J. K. Nurminen, "TCP receive buffer aware wireless multimedia streaming: An energy efficient approach," in Proc. ACM Workshop Netw. Operating Syst. Support Dig. Audio Video, Feb. 2013, pp. 13-18.

[20] M. A. Hoque, M. Siekkinen, and J. K. Nurminen, “On the energy efficiency of proxy-based traffic shaping for mobile audio streaming," in Proc. IEEE Consumer Commun. Netw. Conf., Jan. 2011, pp. 891-895.

[21] J. Korhonen and Y. Wang, "Power-efficient streaming for mobile terminals," in Proc. ACM Workshop Netw. Operating Syst. Support Dig. Audio Video, Jun. 2005, pp. 39-44.

[22] A. Shokrollahi, "Raptor codes," IEEE Trans. Inf. Theory, vol. 52, no. 6, pp. 2551-2567, Jun. 2006.

[23] C. Hsu, C. Fan, T. Tsai, C. Huang, C. Hsu, and K. Chen, "Toward adaptive screencast platform: measurement and optimization," ACM Trans. Multimedia Comput. Commun. Appl., vol. 7, no. 3, Aug. 2015.

[24] S. Chandra, J. Boreczky, and L. Rowe, "High performance manyto-many Intranet screen sharing with DisplayCast," ACM Trans. Multimedia Comput., Commun. Appl., vol. 10, no. 2, Feb. 2014.

[25] C. Zhang, X. Zhang, and R. Chandra, "Energy efficient wiFi display," in Proc. ACM Conf. Mobile Syst. Appl. Services, Nov. 2015, pp. 405-418.

[26] H. Ha, P. Bae, K. Lim, J. Ko, and Y. Ko, "Poster Abstract: Mobile contents on the big screen: Adaptive frame filtering for mobile device screen sharing," in Proc. ACM Conf. Embedded Netw. Sensor Syst., Nov. 2014.

[27] P. Bae, J. Ko, J. Ryu, and Y. Ko, "Poster abstract: Screen dynamics analysis-based adaptive frame skipping for efficient mobile screen sharing," in Proc. ACM Conf. Inf. Process. Sens. Netw., Apr. 2015.

[28] N. Choi, Y. Seok, T. Kwon, and Y. Choi, "Leader-based multicast service in IEEE $802.11 v$ networks," in Proc. IEEE Consumer Commun. Netw. Conf., Jan. 2010, pp. 1-5.

[29] Y. Park, C. Jo, S. Yun, and H. Kim, "Multi-room IPTV delivery through pseudo-broadcast over IEEE 802.11 links," in Proc. IEEE Vehicular Technol. Conf. Spring, May 2010, pp. 1-5.

[30] H.-T. Chiao, S.-Y. Chang, K.-M. Li, Y.-T. Kuo, and M.-C. Tseng, "WiFi multicast streaming using AL-FEC inside the trains of high-speed rails," in Proc. IEEE Int. Symp. Broadband Multimedia Syst. Broadcasting, Jun. 2012, pp. 1-6.

[31] M. Choi, W. Sun, J. Koo, and S. Choi, "Reliable video multicast over Wi-Fi networks with coordinated multiple APs," in Proc. IEEE Conf. Comput. Commun., Apr. 2014, pp. 424-432.

[32] M. Siekkinen, M. A. Hoque, J. K. Nurminen, and M. Aalto, "Streaming over 3G and LTE: how to save smartphone energy in radio access network-friendly way," in Proc. ACM Workshop Mobile Video, Feb. 2013, pp. 13-18.

[33] H. Shen and Q. Qiu, "User-aware energy efficient streaming strategy for smartphone based video playback applications," in Proc. Des. Autom. Test Europe Conf. Exhib., Mar. 2013, pp. 258-261.

[34] C. Poellabauer and K. Schwan, "Energy-aware traffic shaping for wireless real-time applications," in Proc. IEEE Real-Time Embedded Technol. Appl. Symp., May 2004, pp. 48-55.

[35] Y. Go, O. C. Kwon, and H. Song, "An energy-efficient HTTP adaptive video streaming with networking cost constraint over wireless networks," IEEE Trans. Multimedia, vol. 17, no. 9, pp. 1646-1657, Sep. 2015.

[36] O. C. Kwon, Y. Go, and H. Song, "An energy-efficient multimedia streaming transport protocol over heterogeneous wireless networks," IEEE Trans. Vehicular Technol., vol. 65, no. 8, pp. 65186531, Aug. 2016.

[37] O. C. Kwon, Y. Go, Y. Park, and H. Song, "MPMTP: Multipath multimedia transport protocol using systematic raptor codes over wireless networks," IEEE Trans. Mobile Comput., vol. 14, no. 9, pp. 1903-1916, Sep. 2015.

[38] J. Huang, F. Qian, A. Gerber, Z. M. Mao, S. Sen, and O. Spatscheck, "A close examination of performance and power characteristics of 4G LTE networks," in Proc. ACM Conf. Mobile Syst. Appl. Services, Jun. 2012, pp. 225-238.

[39] ARM NEON. [Online]. Available: http://www.arm.com/ products/processors/technologies/neon.php/, Accessed on: 2017.

[40] GStreamer. [Online]. Available: https://gstreamer.freedesktop. org/, Accessed on: 2017.

[41] ODROID. [Online]. Available: http://www.hardkernel.com/ main/main.php/, Accessed on: 2017.
[42] YouTube, A little girl MV. [Online]. Available: https://www. 1107 youtube.com/watch? $\mathrm{v}=\mathrm{bLoO0FSXncg/,} \mathrm{Accessed} \mathrm{on:} 2017$.

[43] Video test sequence. [Online]. Available: http://cs-nsl-wiki.cs. 1109 surrey.sfu.ca/wiki/Video Library and Tools/, Accessed on: 1110 2017.

[44] H. R. Oh, D. O. Wu, and H. Song, "An effective mesh-pull-based 1112 P2P video streaming system using fountain codes with variable 1113 symbol sizes," Comput. Netw., vol. 55, no. 12, pp. 2746-2759, 1114 Aug. 2011

[45] I. S. Reed and G. Solomon, "Polynomial codes over certain finite 1116 fields," J. Soc. Ind. Appl. Math., vol. 8, no. 2, pp. 300-304, 1960.

[46] P. C. Hansen, V. Pereyra and G. Scherer, Least Squares Data Fitting 1118 with Applications. Baltimore, MD, USA: Johns Hopkins Univ. 1119 Press, 2012.

[47] A. Howard, Elementary Linear Algebra. Hoboken, NJ, USA: Wiley, 112 2010.

[48] Linux Traffic Control. [Online]. Available: http://tldp.org/ 1123 HOWTO/Traffic-Control-HOWTO/index.html, Accessed on: 1124 2017.

1125

[49] Power Profiles for Android. [Online]. Available: https://source. 1126 android.com/devices/tech/power/, Accessed on: 2017.

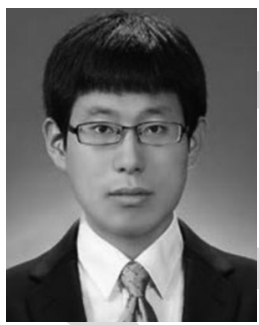

Yunmin Go received the BS degree from the 1128 School of Computer Science and Electrical Engi- 1129 neering, Handong Global University, Korea, in 1130 August 2010. Currently, he is working toward the 1131 $\mathrm{PhD}$ degree in the Division of IT Convergence and 1132 Engineering, Pohang University of Science and 1133 Technology (POSTECH), Korea. His research 1134 interests are in the areas of HTTP adaptive stream- 1135 ing, channel coding-based streaming, and energy- 1136 efficient wireless networks.

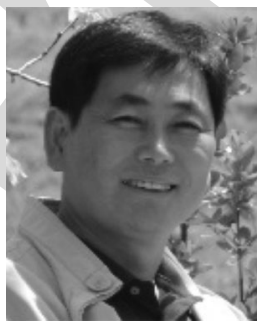

Hwangjun Song received the BS and MS 1138 degrees from the Department of Control and 1139 Instrumentation (EE), Seoul National University, 1140 Korea, in 1990 and 1992, respectively, and the 1141 $\mathrm{PhD}$ degree in electrical engineering-systems 1142 from the University of Southern California, Los 1143 Angeles, CA, USA, in 1999. From 1995 to 1999, 1144 he was a research assistant in SIPI (Signal and 1145 Image Processing Institute) and IMSC (Inte- 1146 grated Media Systems Center), University of 1147 Southern California. From 2000 to 2005, he was 1148 an assistant professor/vice dean of admission affairs at Hongik Univer- 1149 sity, Seoul, Korea. Since Feb. 2005, he has been in the Department of 1150 Computer Science and Engineering, POSTECH (Pohang University of 1151 Science and Technology), Korea. He received the Haedong Paper 1152 Award from the Korean Institute of Communication Science in 2005. He 1153 was a courtesy associate professor with the University of Florida in 1154 2011-2012. He served as a vice president of the Korean Institute of Infor- 1155 mation Scientists and Engineers, in 2016-2017. He is an APSIPA distin- 1156 guished lecturer for 2017-2018. He is an editorial board member of the 1157 Journal of Visual Communication and Image Representation and an 1158 associate editor of the Journal of Communications and Networks, and 1159 served as an editorial board member of the International Journal of 1160 Vehicular Technology and a guest editor of the special issue on network 1161 technologies for emerging broadband multimedia services in the Journal 1162 of Visual Communication and Image Representation and the special 1163 issue on "wireless \& mobile networks" in the International Journal of Ad 1164 Hoc and Ubiquitous Computing. His research interests include multime- 1165 dia signal processing and communication, image/video compression, 1166 digital signal processing, network protocols necessary to implement 1167 functional image/video applications, and control system.

1168

$\triangleright$ For more information on this or any other computing topic, 1169 please visit our Digital Library at www.computer.org/publications/dlib. 1170 